\title{
Effects of Background Noise on the Speech Acoustics of People With Aphasia
}

\author{
Kirsten Dixon \\ Brigham Young University
}

Follow this and additional works at: https://scholarsarchive.byu.edu/etd

Part of the Education Commons

\section{BYU ScholarsArchive Citation}

Dixon, Kirsten, "Effects of Background Noise on the Speech Acoustics of People With Aphasia" (2021). Theses and Dissertations. 9224.

https://scholarsarchive.byu.edu/etd/9224

This Thesis is brought to you for free and open access by BYU ScholarsArchive. It has been accepted for inclusion in Theses and Dissertations by an authorized administrator of BYU ScholarsArchive. For more information, please contact ellen_amatangelo@byu.edu. 
Effects of Background Noise on the Speech Acoustics of People With Aphasia

\title{
Kirsten Dixon
}

\author{
A thesis submitted to the faculty of \\ Brigham Young University \\ in partial fulfillment of the requirements for the degree of \\ Master of Science
}

Christopher Dromey, chair

Tyson G. Harmon

Tracianne Neilsen

\author{
Department of Communication Disorders \\ Brigham Young University
}

Copyright (C) 2021 Kirsten Dixon

All Rights Reserved 


\author{
ABSTRACT \\ Effects of Background Noise on the Speech Acoustics of People With Aphasia \\ Kirsten Dixon \\ Department of Communication Disorders, BYU \\ Master of Science
}

This study investigated the effect of hearing six background noise conditions (silent baseline, pink noise, monologue, lively conversation, one-sided phone call, and cocktail noise) on acoustic measures of speech production during story retells in people with aphasia. Eleven individuals with aphasia and 11 age- and gender-matched control participants took part in the study. Participants heard the background noise conditions through open-back headphones while they retold six short stories. The examiner calculated mean and standard deviation of intensity, mean and standard deviation of fundamental frequency $\left(\mathrm{F}_{0}\right)$, and speech rate in words per minute. A Matlab application that identified pauses (i.e., periods of silence greater than $200 \mathrm{~ms}$ ) computed a speaking time ratio measure (i.e., time speaking versus time pausing). With the exception of the monologue and one-sided phone call condition, both people with aphasia and control participants significantly increase their intensity and $\mathrm{F}_{0}$ in the presence of background noise. Additionally, participants with aphasia have significantly lower speaking time ratios and speaking rates when compared to control participants. Participants make acoustic changes while hearing background noise; speech intensity rises in an effort to increase the signal-to-noise ratio, while mean $\mathrm{F}_{0}$ increases due to a presumed rise in subglottal pressure. Further research is suggested to investigate other acoustic differences, possibly at the segmental level, between speech produced in informational and energetic background noise.

Keywords: aphasia, selective attention, speech acoustics, informational noise, energetic noise 


\section{ACKNOWLEDGEMENTS}

I am grateful for everyone who made this project possible, even during a world-wide pandemic. I am thankful for Dr. Dromey for being an excellent thesis advisor and for demonstrating unwavering patience, flexibility, and support throughout this project. I would also like to thank Dr. Harmon and Dr. Neilsen for taking time out of their busy schedules to provide feedback on my thesis.

I am grateful for Brenna Nelson for playing a huge role in organizing this project, even as she was busy juggling so many responsibilities with her new twin babies. I am also extremely thankful for the participants who sacrificed their time to make this project possible.

I am so appreciative of my family, especially my new husband Sam, who provided endless encouragement throughout this process. I truly have the best people in my life.

Last but not least, I am grateful for the grant from the McKay School of Education that made it possible for both examiners and participants to be reimbursed for their efforts on this project. 


\section{TABLE OF CONTENTS}

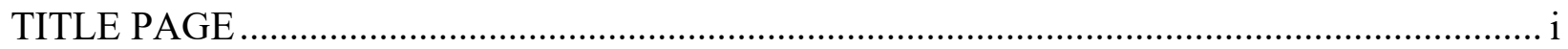

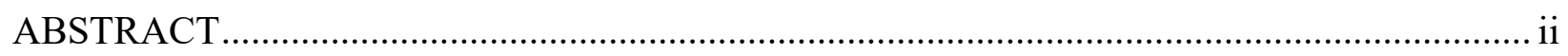

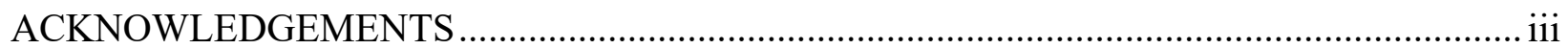

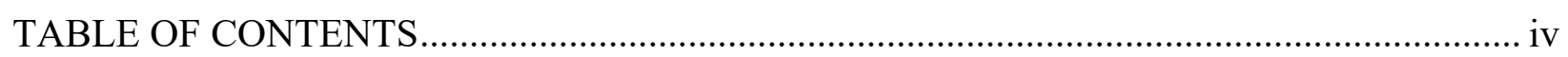

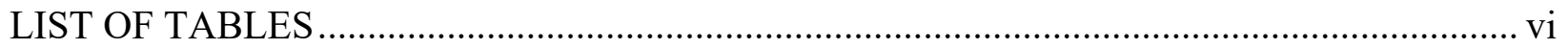

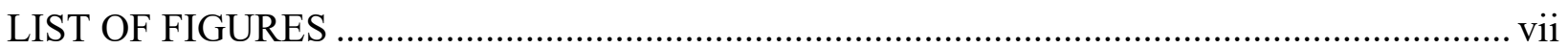

DESCRIPTION OF THESIS STRUCTURE AND CONTENT ........................................... viii

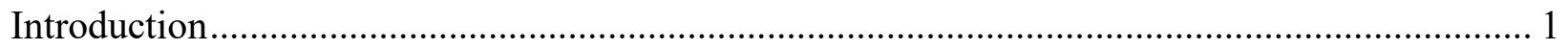

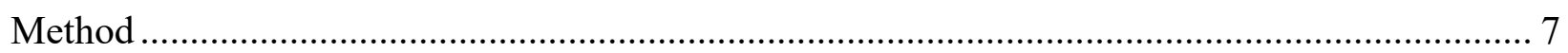

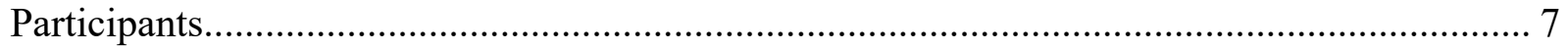

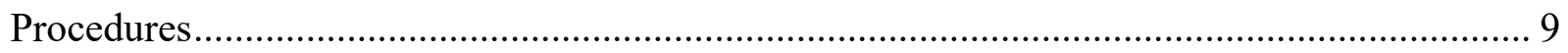

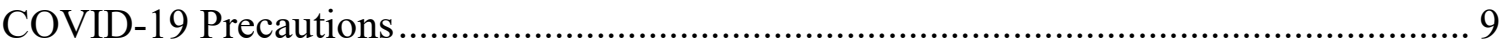

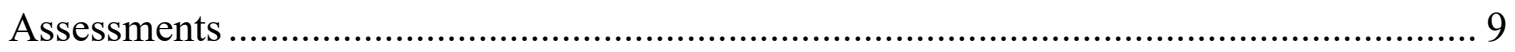

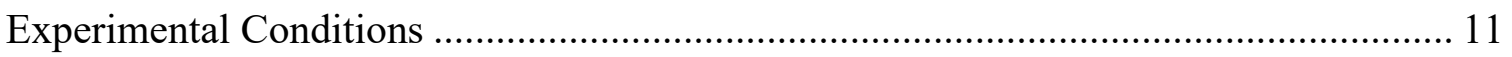

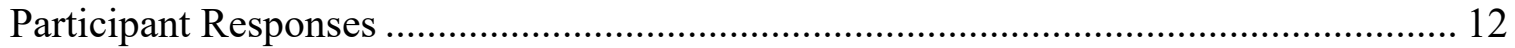

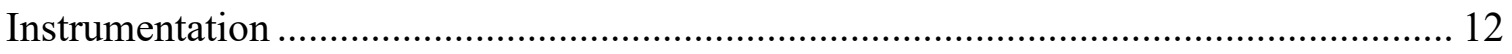

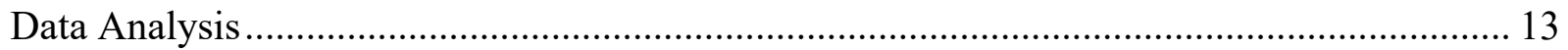

Acoustic Parameters....................................................................................... 13

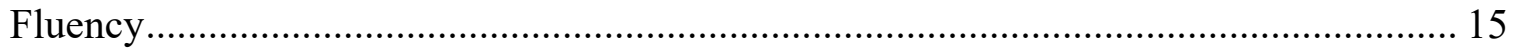

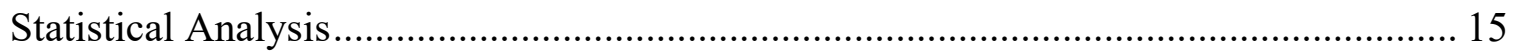

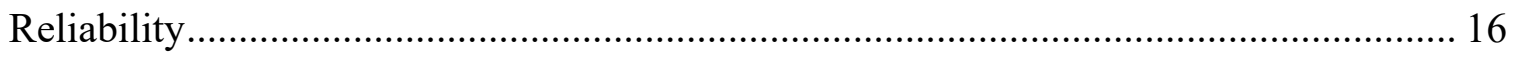

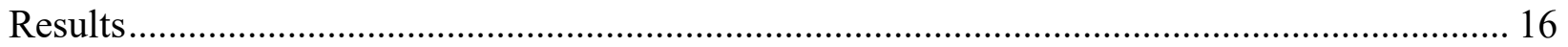




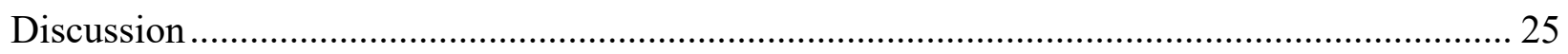

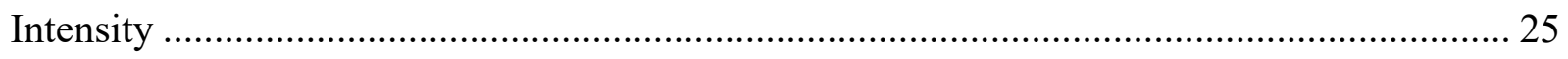

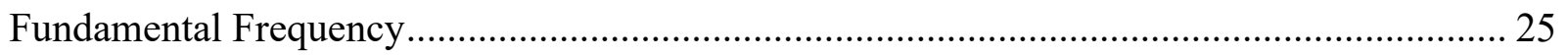

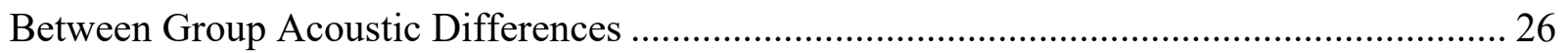

Informational Verses Energetic Background Conditions ..................................................... 27

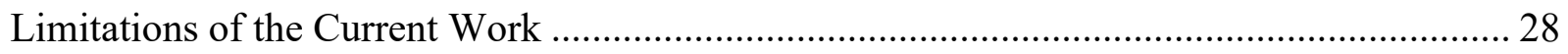

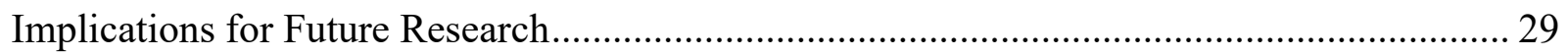

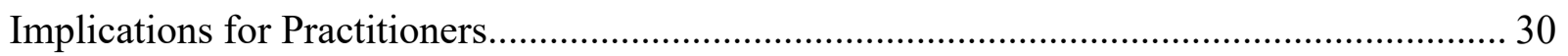

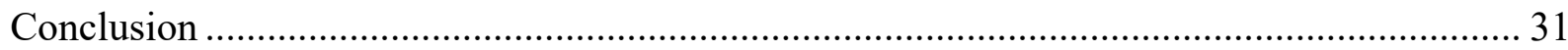

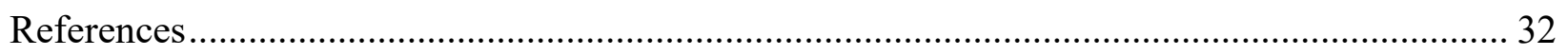

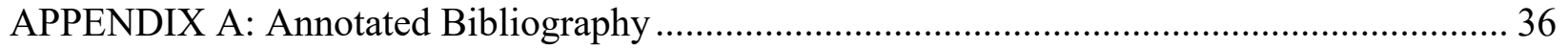

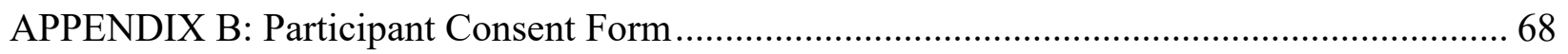




\section{LIST OF TABLES}

Table 1 Demographic Characteristics of the Participants With Aphasia ................................ 8

Table 2 Demographic Characteristics of the Control Participants....................................... 8

Table 3 Quick Aphasia Battery Scores and Hearing Thresholds for Participants With

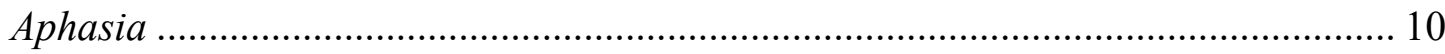

Table 4 Stroke-Free Status Scores and Hearing Thresholds for Control Participants ............ 11

Table 5 Means and Standard Deviations for the Acoustic Measures Across Silent Baseline

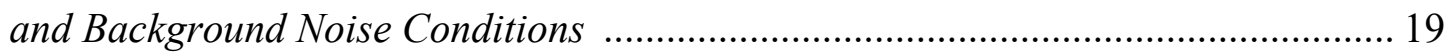

Table 6 ANOVA Main Effects for the Acoustic Measures Across Background Noise

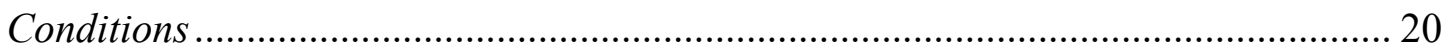

Table 7 Concurrent Contrast Results for Changes in the Acoustic Measures for Individual Background Noise Conditions When Compared to Silent Baseline .......................... 21

Table 8 ANOVA Results for Between Subjects (Control Participants Versus Participants With Aphasia) Effects on Acoustic Measures 


\section{LIST OF FIGURES}

Figure 1 Praat Fo Contours Before and After Adjustments ................................................. 14

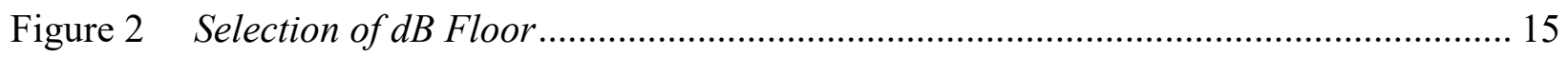

Figure 3 Mean and Standard Deviation of Intensity Across Conditions by Group .................. 23

Figure 4 Mean and Standard Deviation of Fundamental Frequency Across Conditions by

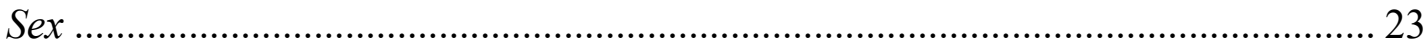

Figure 5 Mean and Standard Deviation of Speaking Time Ratios by Group ......................... 24

Figure 6 Mean and Standard Deviation of Speaking Rates by Group .................................. 24 


\section{DESCRIPTION OF THESIS STRUCTURE AND CONTENT \\ Effects of Background Noise on the Speech Acoustics of People With Aphasia}

is written in a hybrid format that follows the thesis requirements for Brigham Young University as well as the structure for peer-reviewed scientific journals. A review of literature in the form of an annotated bibliography is found in Appendix A. Appendix B consists of a consent form signed by participants prior to completing data collection sessions; this form was approved by the Institutional Review Board. 


\section{Introduction}

Background noise, whether it be the buzz of a fan, noisy chatter in a restaurant, or a nearby person talking on the phone, is part of everyday life. Although people may not always consciously recognize the occurrence of background noise, previous researchers have determined that background noise affects various aspects of life. For example, one study found that employees working in noisy, open office spaces (rather than smaller, quieter environments) reported higher levels of workplace annoyance and increased difficulty working (Di Blasio et al., 2019). Additionally, background noise significantly impacts social interactions, specifically for people with preexisting language deficits.

After sustaining brain injuries, individuals may acquire expressive, receptive, or mixed language deficits that may affect all language-related activities (e.g., participating in a conversation, reading, and writing). This acquired language disorder is referred to as aphasia (Hallowell, 2017, p. 4). A previous qualitative study reported that while being interviewed, 6 out of 21 people with aphasia (PWA) described background noise as a significant communication barrier. These six PWA also mentioned that at times they completely refrained from participating in interactions because of background noise (Harmon, 2020). The current study aims to quantitatively examine how individuals' speech acoustics are affected by various background noise conditions. Previous researchers have determined that dividing one's attention while speaking influences speech movements (Dromey \& Benson, 2003). However, little research has

been performed specifically examining the effects of noise distractions on spoken language in PWA and individuals in a control group as they are exposed to various background noise conditions. 
In an effort to prioritize incoming sensory stimuli (including background noise), people must attend to relevant aspects of the external world. Johnson and Jacobson (2017) described attention as "the ability to maintain a coherent line of thought or action" (p. 31). Supporting these organized thoughts and actions is a "a finite set of brain processes that can interact, mutually and with other brain processes, in the performance of different perceptual, cognitive, and motor tasks" (Parasuraman, 1998, p. 3). Due to the dynamic nature of everyday experiences, humans are equipped with the ability to attend to incoming stimuli in different ways. Cognitive attention is frequently divided into the following four categories: sustained attention, selective attention, divided attention, and alternating attention (Johnson \& Jacobson, 2017). As selective attention aids speakers in effectively communicating in the presence of background noise, selective attention will be discussed in more detail.

Selective attention is used as a filtering mechanism. As individuals are presented with both relevant and distracting stimuli, selective attention aids in maintaining a focus on the intended signals. This is achieved by inhibiting concentration on competing, peripheral stimuli (Johnson \& Jacobson, 2017). Specifically, in the current work participants used selective attention to complete story retell tasks while filtering out irrelevant background noises.

Historically, aphasia has been classified as purely a language disorder (Hallowell, 2017). However, recent studies suggest that cognitive deficits (such as impaired attention) impact the spoken language of PWA (Murray, 2012; Murray et al., 1998). For instance, in Murray's 2012 study, 39 participants with varying severities of aphasia and 39 neurotypical participants with similar ages and education levels completed a cognitive test battery. One of the evaluations included in the battery was the Elevator Counting with Distraction (ECD) subtest of the Test of Everyday Attention (TEA). The ECD evaluated participants' selective attention skills, as the 
participants were required to perform numerical tasks while simultaneously tuning out auditory distractors. Because a wide range of aphasia severity levels were included in the study, scores on the test battery varied among the PWA. However, an independent $t$-test demonstrated that the ECD scores of the participants with aphasia were significantly lower than the control speakers' scores. These results suggest that in addition to language problems, PWA may be liable to attention deficits, including selective attention weaknesses (Murray, 2012; Murray et al., 1998).

Regardless of a person's neurological status, all types of noise can potentially be distracting. However, researchers have found that certain background noise conditions divert attention more than others. Past studies have specifically dealt with two subsets of background noise: energetic masking and informational masking. These types of masking differ in their content. Energetic masking (e.g., pink noise) contains spectral energy that overlaps with the main acoustic signal. This overlapping may result in the masking competing with or overpowering meaningful elements of the main acoustic signal (Lidestam et al., 2014). For instance, a mother may experience difficulty understanding her child while blow drying her hair, as the noise energy produced by the blow dryer may drown out the acoustic elements of the child's message.

On the other hand, informational masking occurs when the background stimuli include linguistic content which overlaps with the intended speech signal. Rather than competing with the spectral aspects of a sound, informational masking serves as a distractor when involuntary processing of irrelevant linguistic information occurs at the same time as comprehension of the main acoustic target (Lidestam et al., 2014). An example of informational masking is a speaker experiencing an inability to fully communicate their thoughts to a friend due to another interesting conversation occurring nearby. Because both the speaker's message and the nearby conversation contain linguistic information, the conversational partners must determine which 
linguistic information is relevant to their own interaction. The effort required to make this distinction increases cognitive demands (Renz et al., 2018).

Previous studies support the notion that informational masking is more cognitively effortful. Researchers found that informational masking decreased visual speechreading accuracy, while energetic masking had no effect on accuracy (Lidestam et al., 2014). Another study determined that when solving arithmetic problems, participants demonstrated the most difficulty accurately solving the problems if the background noise presented inaccurate, but linguistically similar solutions. For instance, noise stimuli containing ascending numbers were the most distracting for participants solving addition problems, as the ascension of digits showed similarities to the process performed when adding numbers together (Perham et al., 2016).

While informational masking increases listeners' cognitive loads, both informational and energetic masking can influence the production of spoken language. In 1911, Etienne Lombard observed that when individuals spoke in noisy environments, the intensity of their speech also increased. This finding, subsequently named the Lombard effect, demonstrated that individuals tend to automatically adjust their vocal output in less-than-ideal communication situations. These adjustments have been explained as an attempt to increase intelligibility (Summers et al., 1988). Summers et al. (1988) performed a study of acoustic changes in the presence of noise. Two adult male participants read 15 stimulus words in varying energetic masking conditions (silence, 80, 90 , and $100 \mathrm{~dB}$ white noise). In addition to observing statistically significant increases in vocal intensity as the participants spoke in noise, the authors found that the two speakers lengthened their words, demonstrated an increase in fundamental frequency $\left(\mathrm{F}_{0}\right)$, and shifted the spectral content of their speech to higher frequencies. 
Other studies have reported similar findings. In both energetic and informational masking conditions, individuals frequently increase vocal effort in order to strengthen the signal-to-noise ratio (Chapman, 2019; Cooke \& Lu, 2010; Dromey \& Scott, 2016; Hazan \& Baker, 2011; Lu \& Cooke, 2008; Smiljanic \& Gilbert, 2017). Additionally, other researchers found that speakers slowed down their speech rate and lengthened the duration of words, thereby creating longer opportunities for listeners to perceive key acoustic information (Lu \& Cooke, 2009a). Lastly, another study reported an increase in $\mathrm{F}_{0}$ and a shift in spectral energy, meaning the spectral energy in the main acoustic signal primarily fell between 1 and $4 \mathrm{kHz}$ (Graetzer et al., 2017; Lu $\&$ Cooke, 2009b). This shift placed sound energy in spectral areas that were less likely to be covered by the masking (Lu \& Cooke, 2009b).

Cooke and $\mathrm{Lu}$ (2010) found that when speakers communicated while simultaneously listening to strong linguistic content (i.e., informational masking), they took advantage of pauses in the stimuli and increased their output of acoustic information during these breaks. Thus, the temporal quality of their speech changed when compared to speech produced in energetic masking and silent conditions. The typical change associated with the Lombard effect is that speakers make acoustic changes to increase intelligibility in noisy circumstances. Certain acoustic changes, however, may increase intelligibility more than others. For example, one study found that while spectral shifting and slower speech rate raised intelligibility, an increase in $\mathrm{F}_{0}$ did not (Lu \& Cooke, 2009a).

As mentioned previously, a recent qualitative study identified background noise as a communication barrier for PWA (Harmon, 2020). After performing interviews with 44 individuals with communication disorders (12 of whom had communication disorders following strokes), Baylor et al. (2011) determined that informational masking conditions were especially 
distracting for stroke survivors, and therefore made it more difficult for them to produce coherent spoken language. The authors also noted that while many intervention approaches focus on identifying and treating particular communication disorders, it could be beneficial for clinicians to put greater emphasis on the environmental and personal factors that influence day to day communication. For example, rather than performing speech-language intervention sessions in quiet clinic rooms, clinicians could focus on potential environmental barriers by presenting distracting noise stimuli during therapy activities. Together, the clinician and client could discuss and practice selective attention strategies to foster improved spoken language skills.

The current work was part of a larger mixed-method study examining the effects of background noise on both expressive language structure, speech production, and communication strategies. The present study focused on the acoustic aspects of speech. Previous research has determined that attentional demands influence the expressive language skills of PWA (Harmon et al., 2019; Murray, 2012; Murray et al., 1998). However, little research has been completed to determine how attentional demands affect the acoustic components of their speech. As acoustic changes could increase the intelligibility of spoken language produced in noise (Lu \& Cooke, 2009a), the current work examined how PWA and their age- and gender-matched peers changed their speech when retelling stories in different background noise conditions.

By presenting both energetic and informational noise stimuli, a further goal was to determine which background noise stimuli evoked the most significant acoustic changes in spoken language. It was reasoned that due to possible selective attention deficits (Murray, 2012), the participants with aphasia would demonstrate greater speech acoustic changes than the speakers in the control group. It was also hypothesized that spoken language produced in informational masking conditions would exhibit greater acoustic changes than in energetic 
masking conditions for both the PWA and control groups (Cooke \& Lu, 2010; Lidestam et al., 2014; Perham et al., 2016).

Speech-language pathology intervention sessions typically occur in quiet rooms with just the clinician and PWA present. As PWA have previously noted the adverse effects of background noise on their communication (Baylor et al., 2011; Harmon, 2020), it is important that further research investigate the changes in speech produced in background noise. The results from this study could be beneficial in guiding clinicians to use both energetic and informational maskers in intervention sessions with PWA to simulate everyday background noises. Together, the clinician and PWA could practice strategies to increase selective attention abilities, and therefore overall communication, in the presence of distracting background noises.

\section{Method}

\section{Participants}

Eleven individuals with mild to moderate aphasia and a control group of 11 adults with no history of neurological damage took part in the study (see Table 1, Table 2). The controls were age- and gender-matched with the PWA. All participants were native English speakers. PWA were recruited by phone calls and emails after voluntarily placing their names and contact information on the Brigham Young University (BYU) Stroke and Brain Injury Registry. In addition, informational fliers with contact details of the experimenters were distributed to PWA receiving services at the BYU Speech and Language Clinic, inpatient and outpatient hospital units, individuals participating in stroke and brain injury support groups, and community members with aphasia. All recruitment processes and experimental procedures were approved by BYU's Institutional Review Board (IRB) in March of 2020. 
Table 1

Demographic Characteristics of the Participants With Aphasia

\begin{tabular}{rccccrc}
\hline ID & Age & Sex & Ed & Etiology & MPO & Lang \\
\hline 01 & 46 & F & 16 & Stroke & 94 & 2 \\
02 & 53 & M & 19 & Stroke & 85 & 2 \\
03 & 69 & M & 18 & Necrosis & 60 & 2 \\
04 & 49 & F & 12 & Stroke & 206 & 1 \\
05 & 44 & M & 14 & TBI & 56 & 1 \\
06 & 35 & M & 15 & Stroke & 131 & 1 \\
07 & 55 & M & 16 & Stroke & 5 & 3 \\
08 & 62 & F & 13 & Stroke & 105 & 1 \\
10 & 47 & M & 18 & Stroke & 252 & 2 \\
11 & 52 & M & 18 & Stroke & 3 & 1 \\
12 & 60 & M & 16 & Stroke & 52 & 2 \\
\hline
\end{tabular}

Note. ID=participant ID; Age (years); Ed=years of education; TBI=traumatic brain injury;

$\mathrm{MPO}=$ months post onset of aphasia at time of session; Lang=number of languages spoken .

\section{Table 2}

Demographic Characteristics of the Control Participants

\begin{tabular}{cccccc}
\hline ID & Match & Age & Sex & Ed & Lang \\
\hline 13 & 02 & 48 & M & 18 & 1 \\
14 & 04 & 53 & F & 16 & 1 \\
15 & 03 & 74 & M & 16 & 2 \\
16 & 05 & 45 & M & 20 & 1 \\
17 & 01 & 44 & F & 17 & 1 \\
18 & 06 & 32 & M & 16 & 2 \\
19 & 10 & 48 & M & 22 & 2 \\
20 & 07 & 56 & M & 18 & 2 \\
21 & 12 & 55 & M & 17 & 2 \\
22 & 11 & 49 & M & 14 & 1 \\
23 & 08 & 57 & F & 18 & 2 \\
\hline
\end{tabular}

Note. ID=participant ID; Match=age- and gender-matched PWA; Age (years);

$\mathrm{Ed}=$ years of education; Lang=number of languages spoken. 


\section{Procedures}

\section{COVID-19 Precautions}

Participants attended one 90 to 120-minute session at the BYU Speech and Language Clinic. Prior to initiating each session, both experimenters and participants were screened for COVID-19 symptoms and had their temperatures checked. In accordance with the IRB's COVID-19 standards, all lab equipment was sanitized before and after sessions, social distancing and face coverings were employed throughout sessions, and a maximum of one session was scheduled per day.

\section{Assessments}

At the start of each session, participants were briefed on experimental procedures and signed a consent form. PWA completed a short, 15-minute standardized assessment, The Quick Aphasia Battery (QAB). In addition to providing information regarding each individual's language profile, the QAB served as a dysarthria and voice screener (Wilson et al., 2018). Control group participants filled out the Questionnaire for Verifying Stroke-Free Status (QVSFS) to ensure that no previous neurological injuries acted as extraneous variables in the experiment (Jones et al., 2001). Hearing thresholds at $0.5 \mathrm{kHz}, 1 \mathrm{kHz}, 2 \mathrm{kHz}$, and $4 \mathrm{kHz}$ were measured for each participant to verify the short stories and background stimuli were easily audible (see Table 3, Table 4). 
Table 3

Quick Aphasia Battery Scores and Hearing Thresholds for Participants With Aphasia

\begin{tabular}{|c|c|c|c|c|c|c|c|c|c|c|c|}
\hline \multirow[b]{2}{*}{ ID } & \multicolumn{9}{|c|}{ Quick Aphasia Battery Scores } & \multirow[b]{2}{*}{ RTA } & \multirow[b]{2}{*}{ LTA } \\
\hline & $\mathrm{WC}$ & $\mathrm{SC}$ & WF & GC & SMP & Rep & Rea & $\mathrm{OA}$ & Sev & & \\
\hline 01 & 9.6 & 10.0 & 10.0 & 10.0 & 10.0 & 10.0 & 10.0 & 9.8 & VM & -1.25 & 1.25 \\
\hline 02 & 10.0 & 7.1 & 6.3 & 8.6 & 7.5 & 7.9 & 8.3 & 8.1 & Mild & 5.00 & 10.00 \\
\hline 03 & 9.6 & 1.7 & 5.0 & 7.0 & 10.0 & 5.4 & 7.1 & 6.4 & Mod & 22.50 & 35.00 \\
\hline 04 & 10.0 & 4.2 & 7.0 & 1.4 & 7.5 & 5.8 & 7.9 & 5.8 & $\mathrm{Mo}$ & 3.75 & 3.75 \\
\hline 05 & 10.0 & 8.3 & 8.0 & 10.0 & 10.0 & 10.0 & 9.6 & 9.3 & VM & 8.75 & 7.50 \\
\hline 06 & 10.0 & 7.5 & 3.5 & 8.4 & 10.0 & 5.8 & 6.3 & 7.5 & Mild & 8.75 & 11.25 \\
\hline 07 & 10.0 & 3.3 & 7.3 & 7.5 & 10.0 & 6.3 & 7.9 & 7.3 & Mod & 1.25 & 7.50 \\
\hline 08 & 10.0 & 4.2 & 3.0 & 4.6 & 5.0 & 5.0 & 2.5 & 5.2 & Mod & 17.50 & 17.50 \\
\hline 10 & 10.0 & 7.1 & 9.0 & 9.1 & 10.0 & 8.8 & 10.0 & 8.9 & VM & 3.75 & 6.25 \\
\hline 11 & 10.0 & 9.6 & 9.0 & 10.0 & 10.0 & 10.0 & 10.0 & 9.8 & VM & 12.50 & 13.75 \\
\hline 12 & 10.0 & 7.1 & 9.0 & 10.0 & 10.0 & 10.0 & 10.0 & 9.2 & VM & 26.25 & 30.00 \\
\hline
\end{tabular}

Note. $\mathrm{ID}=$ participant ID; WC=Word Comprehension; $\mathrm{SC}=$ Sentence Comprehension; WF=Word

Finding; GC=Grammatical Construction; SMP=Speech Motor Programming; Rep=Repetition;

Rea=Reading; $\mathrm{OA}=$ Overall; Sev=Severity; $\mathrm{VM}=$ =very mild; $\mathrm{Mod}=$ moderate; $\mathrm{RTA}=$ Right Hearing Threshold Average (dB HL); LTA=Left Hearing Threshold Average (dB HL). 


\section{Table 4}

Stroke-Free Status Scores and Hearing Thresholds for Control Participants

\begin{tabular}{ccrr}
\hline ID & QVSFS & RTA & LTA \\
\hline 13 & 0 & 8.75 & 23.75 \\
14 & 0 & 20.00 & 18.75 \\
15 & 0 & 31.25 & 43.75 \\
16 & 0 & 8.75 & 8.75 \\
17 & 0 & 3.75 & 7.50 \\
18 & 0 & 7.50 & 5.00 \\
19 & 0 & 6.25 & 6.25 \\
20 & 0 & 8.75 & 17.50 \\
21 & 0 & 5.00 & 0.00 \\
22 & 0 & 30.00 & 33.75 \\
23 & 0 & 7.50 & 2.50 \\
\hline
\end{tabular}

Note. ID=Participant ID; QVSFS=Questionnaire for Verifying Stroke-Free

Status; RTA=Right Hearing Threshold Average (dB HL); LTA=Left

Hearing Threshold Average (dB HL).

\section{Experimental Conditions}

During the experiment, participants listened to seven short stories in silence. They then retold the stories under different noise conditions. The first story was retold in silence to allow participants to practice the story retell task and experimenters to check all lab equipment. Participants proceeded to retell the remaining stories while being exposed to the six background noise conditions. Both the order of the stories and the background noise stimuli were randomized. The background noise stimuli consisted of the following six conditions: silent baseline, pink noise, a monologue, a one-sided phone call, a lively conversation between multiple speakers, and six-speaker cocktail speech. To determine the intensity at which the background noises were presented, the experimenters perceptually matched the pink noise stimulus to $60 \mathrm{~dB}$ HL masking noise from an audiometer. The mean intensity of the pink noise 
was then used as a reference point to equalize the remainder of the background noise stimuli using Audacity (Audacity Team, 2020).

\section{Participant Responses}

Participants answered six questions to rate how they felt during each retell. The questionnaire involved the following themes: perceived success, level of effort, amount of stress, and whether or not participants felt pleasant, nervous, or calm during retells. Questions were answered on a scale of one to five (one corresponding with "not at all," five corresponding with "extremely"). After completing the experimental condition, participants were interviewed on which background noise stimuli they found the least or most distracting, general observations throughout the experiment, strategies they used to focus on the story retell task, and if any part of the experiment reminded them of everyday communication situations involving background noise. The results from the questionnaires and interviews will be analyzed and reported in a future study.

\section{Instrumentation}

The hearing threshold test and experiment were conducted in a sound-attenuating booth to limit sound interference. Participants wore open back headphones in order to avoid disruption of the auditory feedback loop, thus allowing them to monitor their speech. A sound level meter was placed $50 \mathrm{~cm}$ from participants' mouths and measured vocal intensity while participants sustained a steady vowel for three seconds. These sound pressure level values were used as points of reference when calculating mean intensity values during subsequent data analysis. Participants retold the short stories with a boom microphone placed approximately $100 \mathrm{~cm}$ from their mouths. Spoken language was digitized using a FocusRite Scarlett 2i2 USB converter and recorded with Adobe Audition (Adobe Inc., 2021). 


\section{Data Analysis}

\section{Acoustic Parameters}

Non-speech sounds (e.g., laughing, coughing, throat clearing, etc.), as well as spoken language that did not relate to the retells, were manually removed from the sound files. Additionally, the examiner performed $\mathrm{F}_{0}$ and intensity analyses using Praat speech analysis software (Boersma \& Weenink, 2021). Praat automatically formed $\mathrm{F}_{0}$ contours by detecting patterns (i.e., vocal fold vibrations) in the sound waveforms. The examiner listened to and viewed $\mathrm{F}_{0}$ artifacts, or areas where $\mathrm{F}_{0}$ differed significantly from surrounding frequency values. Artifacts that were not true vocal fold vibration (e.g., vocal fry and fricatives) were manually removed from $\mathrm{F}_{0}$ contours (see Figure 1). A customized Matlab application used the adjusted $\mathrm{F}_{0}$ contours to compute the mean and standard deviation of $\mathrm{F}_{0}$ (measured in $\mathrm{Hz}$ ). To account for the inherent differences in $\mathrm{F}_{0}$ between males and females, the standard deviations in $\mathrm{Hz}$ were converted into semitones with a spreadsheet equation. In order to avoid pauses in the speech samples from affecting the mean intensity, values above a dB floor value that corresponded to the softest speech sounds were used (see Figure 2). 


\section{Figure 1}

Praat $F_{0}$ Contours Before and After Adjustments
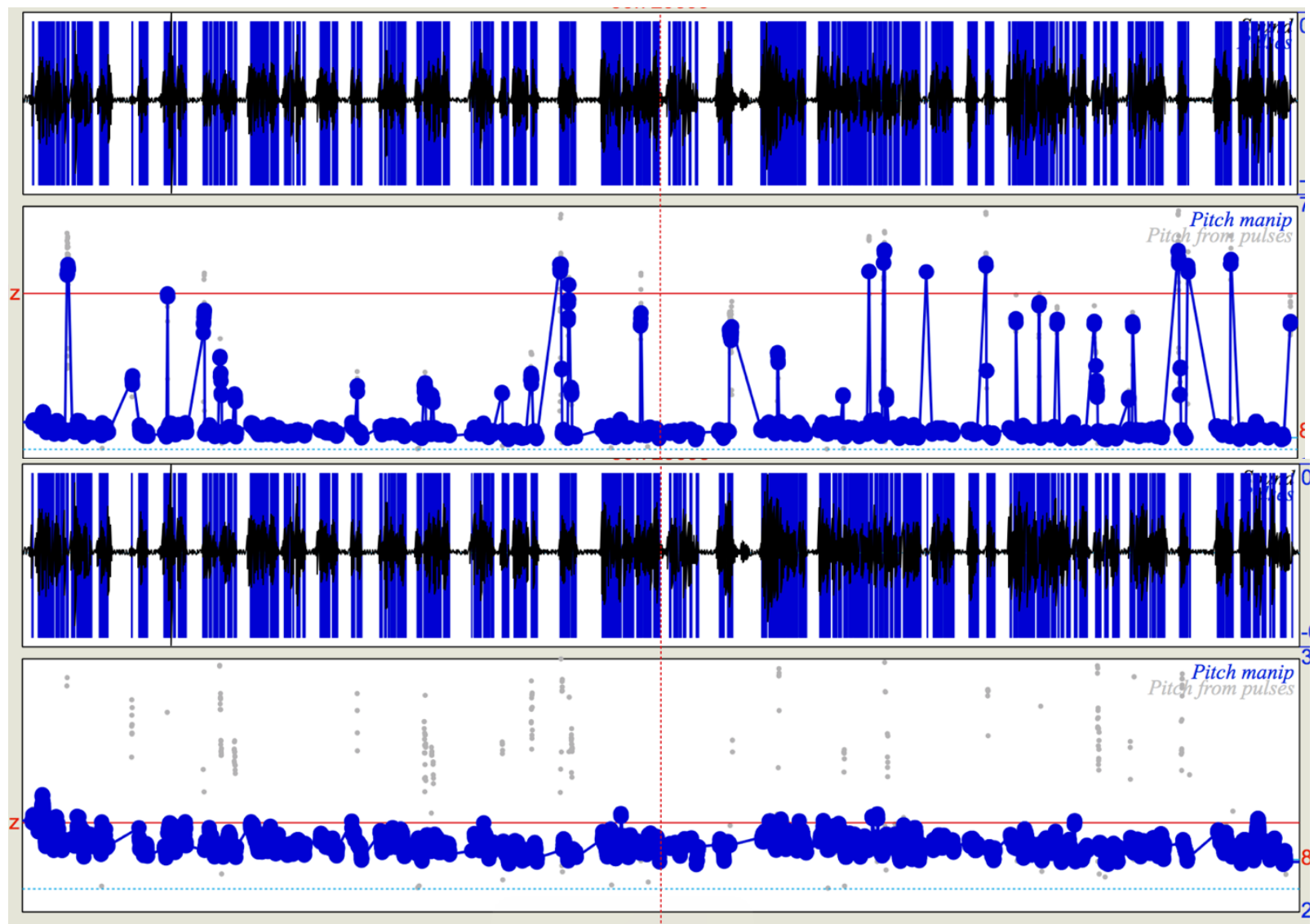

Note. The examiner listened to artifacts (shown as vertical lines with blue dots in the upper contour) and compared them to the waveform to determine if they represented true vocal fold vibration. Artifacts that misrepresented vocal fold vibration were manually removed prior to calculating mean and standard deviation of $\mathrm{F}_{0}$. The contour below does not contain artifacts and is, therefore, a better representation of the participant's actual $\mathrm{F}_{0}$ throughout the story retell. 


\section{Figure 2}

Selection of $d B$ Floor

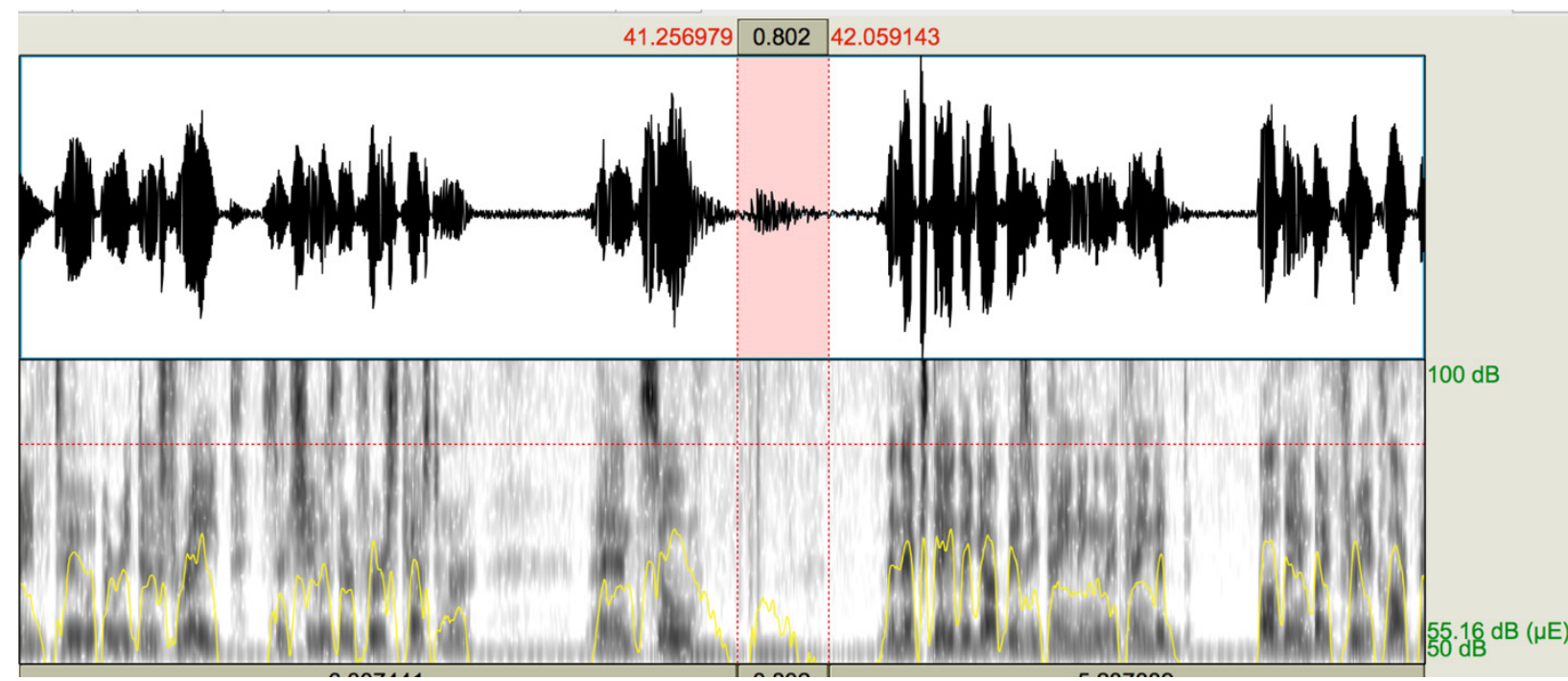

Note. The highlighted area of the waveform corresponds with the softest speech present in the sample. The yellow intensity contours and corresponding $\mathrm{dB}$ values (shown to the right in green) were used to determine a dB floor.

\section{Fluency}

Pauses (i.e., silent periods over $200 \mathrm{~ms}$ ) were identified by a custom Matlab application to calculate speaking time ratio (amount of time a participant was speaking vs. pausing). Orthographic transcriptions (transcribed for language analyses) were used to measure speaking rate in words per minute.

\section{Statistical Analysis}

Repeated measure ANOVAs were performed on the acoustic measures, with significance corresponding to a $p$-value of .05 or less. ANOVA results were interpreted with the assumption of sphericity (Mauchly's sphericity test). If the sphericity assumption was violated (i.e., a Mauchly test $p$ value at or less than .05), a Huynh-Feldt correction was applied to the results. The main analysis tested for changes across the silent baseline and the five background noise 
conditions. along with any interactions with group (PWA versus control). A concurrent contrast analysis tested for differences in the acoustic measures for individual background noise conditions compared with the silent condition.

\section{Reliability}

Participant identification numbers were sorted with a random number generator. Two participants were randomly selected (13 and 22). All acoustic measures were recalculated for the sound files from these speakers. To check for reliability, the new acoustic measures were compared to the original acoustic measures. The correlation between the new and original measures was $\mathrm{r}=.986$.

\section{Results}

The purpose of this study was to determine how PWA and control participants change their speech production in the presence of both informational and energetic noise. Descriptive statistics for the acoustic measures in the silent baseline and five background noise conditions are reported in Table 5. Participant 06's data were not included in the statistical analyses because he only completed four of the six experimental conditions. Additionally, participant 21 's intensity data were excluded from statistical analyses due to technical errors that affected the validity of recorded intensities.

There were significant changes in mean intensity, $F(3.257,58.619)=17.54, p<.001$, ES .494 and mean $\mathrm{F}_{0}, F(5,95)=5.69, p<.001$, ES .23 across the noise conditions (see Table 6, Figure 3, Figure 4). No significant differences in semitone standard deviation, standard deviation of intensity, speaking time ratio, or rate were found. 
Contrast analyses revealed that all conditions exhibited significant increases in mean intensity (pink, $F(1,18)=23.083, p<.001$, ES .562, monologue, $F(1,18)=20.435, p<.001$, ES .532 , phone call, $F(1,18)=19.979, p<.001$, ES .526 , lively conversation, $F(1,18)=32.317, p<$ .001 , ES .642, and cocktail speech, $F(1,18)=38.306, p<.001$, ES .68). Mean $\mathrm{F}_{0}$ produced in pink noise, $F(1,19)=13.014, p=.002$, ES .407, during the lively conversation, $F(1,19)=18.521, p<$ .001 , ES .494 , and in cocktail speech, $F(1,19)=10.018, p=.005$, ES .345 were significantly higher, while mean $\mathrm{F}_{0}$ produced during the monologue and phone call conditions did not demonstrate significant differences (see Table 7). In addition, no significant changes in semitone standard deviation, standard deviation of intensity, speaking time ratio, and rate occurred across conditions when compared to silent baseline (see Table 7, Figure 3, Figure 4).

A between-subjects test revealed significant differences between the participants with aphasia and the control participants for speaking time ratio, $F(1,19)=17.544, p<.001$, ES .48 and rate, $F(1,19)=17.06, p=.001$, ES .473 . Both measures were significantly lower for PWA. Additionally, mean intensity, $F(1,18)=3.438, p=.08$, ES .16 showed a non-significant trend of higher intensity for individuals with aphasia. No differences were found in mean $\mathrm{F}_{0}$, semitone standard deviation, and standard deviation of intensity between groups (see Table 8, Figure 5, Figure 6).

Lastly, correlations were computed between several participant variables (including Quick Aphasia Battery (QAB) test scores for PWA) and acoustic metrics. Pure tone average for both ears showed a moderate, positive correlation with age, $r(22)=.607, p=.003$, and speaking rate produced during the silent baseline condition showed a moderate, positive correlation with years of education, $r(22)=.538, p=.01$. In participants with aphasia, speaking time ratio was moderately, positively correlated with Overall QAB scores, $r(11)=.647, p=.031$. Rate 
demonstrated a strong, positive correlation with Grammatical Construction, $r(11)=.775, p=.005$, Speech Motor Programming, $r(11)=.785, p=.004$, and Overall QAB scores, $r(11)=.728, p<$ .001 . 
Table 5

Means and Standard Deviations for the Acoustic Measures Across Silent Baseline and Background Noise Conditions

\begin{tabular}{|c|c|c|c|c|c|c|c|c|c|c|c|c|c|}
\hline \multirow[b]{2}{*}{ Measure } & \multirow[b]{2}{*}{ Group } & \multicolumn{12}{|c|}{ Condition (Measure) } \\
\hline & & SL (M) & $\mathrm{SL}(\mathrm{SD})$ & $\mathrm{P}(\mathrm{M})$ & $\mathrm{P}(\mathrm{SD})$ & $\mathrm{M}(\mathrm{M})$ & $\mathrm{M}(\mathrm{SD})$ & $\mathrm{PC}(\mathrm{M})$ & $\mathrm{PC}(\mathrm{SD})$ & LC (M) & LC (SD) & $\mathrm{CT}(\mathrm{M})$ & $\mathrm{CT}(\mathrm{SD})$ \\
\hline \multirow[t]{2}{*}{ Mean dB } & Control & 60.6 & 3.2 & 63.5 & 3.0 & 62.6 & 3.3 & 62.3 & 3.0 & 62.9 & 3.4 & 63.8 & 3.1 \\
\hline & PWA & 64.5 & 4.6 & 66.4 & 4.8 & 65.8 & 5.1 & 65.3 & 4.1 & 66.3 & 5.4 & 67.4 & 5.3 \\
\hline \multirow[t]{2}{*}{$\mathrm{SD} d \mathrm{~B}$} & Control & 4.6 & 1.3 & 4.9 & 0.9 & 4.8 & 1.0 & 4.7 & 0.9 & 4.6 & 0.7 & 4.9 & 0.8 \\
\hline & PWA & 4.9 & 1.2 & 5.0 & 0.9 & 5.1 & 1.2 & 4.9 & 0.9 & 5.5 & 1.0 & 5.3 & 0.9 \\
\hline \multirow[t]{2}{*}{ Mean $\mathrm{F}_{0}$} & Male & 115.8 & 20.9 & 122.8 & 21.4 & 120.6 & 22.7 & 118.3 & 19.9 & 121.7 & 20.4 & 123.1 & 21.4 \\
\hline & Female & 179.9 & 30.3 & 187.5 & 31.0 & 182.5 & 29.5 & 181.7 & 31.6 & 188.7 & 34.4 & 185.3 & 29.7 \\
\hline \multirow[t]{2}{*}{ ST SD } & Control & 2.6 & 0.8 & 2.5 & 0.7 & 2.6 & 0.8 & 2.5 & 0.6 & 2.6 & 0.6 & 2.4 & 0.5 \\
\hline & PWA & 3.0 & 1.3 & 2.7 & 1.1 & 2.7 & 1.1 & 2.9 & 1.0 & 2.6 & 0.9 & 2.9 & 1.3 \\
\hline \multirow[t]{2}{*}{ STR } & Control & .6 & .1 & .7 & .1 & .7 & .1 & .7 & .1 & .7 & .1 & .7 & .1 \\
\hline & PWA & .5 & .1 & .5 & .2 & .5 & .2 & .5 & .1 & .5 & .1 & .5 & .1 \\
\hline \multirow[t]{2}{*}{ Rate } & Control & 158.0 & 23.0 & 150.0 & 21.7 & 154.5 & 21.9 & 156.1 & 23.6 & 149.1 & 19.7 & 151.0 & 21.9 \\
\hline & PWA & 101.9 & 37.9 & 101.8 & 36.1 & 107.9 & 42.3 & 105.9 & 35.9 & 98.7 & 28.4 & 103.4 & 35.6 \\
\hline
\end{tabular}

Note. Mean $\mathrm{dB}=$ mean intensity $(\mathrm{dB}) ; \mathrm{SD} d \mathrm{~dB}=$ standard deviation of mean intensity $(\mathrm{dB})$; Mean $\mathrm{F}_{0}(\mathrm{~Hz})$; $\mathrm{ST} \mathrm{SD}=$ semitone standard deviation; $\mathrm{STR}=$ speaking time ratio; Rate (words per minute); $(\mathrm{M})=$ =mean; $(\mathrm{SD})=$ standard deviation; $\mathrm{SL}=$ silent baseline; $\mathrm{P}=$ =pink noise; $\mathrm{M}=$ monologue; $\mathrm{PC}=$ phone call; $\mathrm{LC}=$ lively conversation; $\mathrm{CT}=$ cocktail noise. Mean $\mathrm{F}_{0}$ is reported in groups by sex to avoid low male and high female $\mathrm{F}_{0}$ values from resulting in non-representative mean $\mathrm{F}_{0}$ values. 


\section{Table 6}

ANOVA Main Effects for the Acoustic Measures Across Background Noise Conditions

\begin{tabular}{ccccc}
\hline & \multicolumn{4}{c}{ ANOVA Results } \\
\cline { 2 - 5 } Measure & $\mathrm{df}$ & F-ratio & $p$ & Effect Size \\
\hline Mean dB & $3.257,58.619$ & 17.540 & $<.001$ & .494 \\
SD dB & 5,95 & 1.372 & 0.263 & .071 \\
Mean F0 & 5,95 & 5.690 & $<.001$ & .230 \\
ST SD & 5,95 & 0.708 & .619 & .036 \\
STR & 5,95 & 0.547 & .740 & .028 \\
Rate & 5,95 & 1.348 & .251 & .066 \\
\hline
\end{tabular}

Note. Mean $\mathrm{dB}=$ mean intensity $(\mathrm{dB}) ; \mathrm{SD} \mathrm{dB}=$ standard deviation of mean intensity $(\mathrm{dB})$; Mean

$\mathrm{F}_{0}(\mathrm{~Hz}) ; \mathrm{ST} \mathrm{SD}=$ semitone standard deviation; $\mathrm{STR}=$ speaking time ratio; Rate (words per minute). 


\section{Table 7}

Concurrent Contrast Results for Changes in the Acoustic Measures for Individual Background Noise Conditions When Compared to Silent Baseline

\begin{tabular}{|c|c|c|c|c|c|c|}
\hline \multirow[b]{2}{*}{ Measure } & \multirow[b]{2}{*}{$\begin{array}{c}\text { ANOVA } \\
\text { Results }\end{array}$} & \multicolumn{5}{|c|}{ Background Noise Condition } \\
\hline & & $\mathrm{P}$ & M & $\mathrm{PC}$ & $\mathrm{LC}$ & $\mathrm{CT}$ \\
\hline \multirow[t]{3}{*}{ Mean dB } & F-ratio & 23.083 & 20.435 & 19.979 & 32.217 & 38.306 \\
\hline & $p$ & $<.001$ & $<.001$ & $<.001$ & $<.001$ & $<.001$ \\
\hline & ES & .562 & .532 & .526 & .642 & .680 \\
\hline \multirow[t]{3}{*}{$\mathrm{SD} d \mathrm{~B}$} & F-ratio & 1.214 & 0.992 & 0.003 & 2.295 & 1.568 \\
\hline & $p$ & .285 & .332 & .956 & .147 & .226 \\
\hline & ES & .063 & .052 & $<.001$ & .113 & .080 \\
\hline \multirow[t]{3}{*}{ Mean $\mathrm{F}_{0}$} & F-ratio & 13.014 & 2.753 & 1.401 & 18.521 & 10.018 \\
\hline & $p$ & .002 & .113 & .251 & $<.001$ & .005 \\
\hline & ES & .407 & .127 & .069 & .494 & .345 \\
\hline \multirow[t]{3}{*}{ ST SD } & F-ratio & 2.05 & 1.048 & 0.395 & 1.230 & 2.325 \\
\hline & $p$ & .168 & .319 & .537 & .281 & .144 \\
\hline & ES & .097 & .052 & .020 & .061 & .109 \\
\hline \multirow[t]{3}{*}{ STR } & F-ratio & 0.603 & 0.824 & 1.218 & 0.052 & 1.147 \\
\hline & $p$ & .447 & .375 & .284 & .823 & .298 \\
\hline & ES & .031 & .042 & .060 & .003 & .057 \\
\hline \multirow[t]{3}{*}{ Rate } & F-ratio & 1.364 & 0.071 & 0.088 & 1.883 & 0.755 \\
\hline & $p$ & .257 & .793 & .770 & .186 & .396 \\
\hline & ES & .067 & .004 & .005 & .090 & .038 \\
\hline
\end{tabular}

Note. Mean $\mathrm{dB}=$ mean intensity $(\mathrm{dB}) ; \mathrm{SD} \mathrm{dB}=$ standard deviation of mean intensity $(\mathrm{dB})$; Mean

$\mathrm{F}_{0}(\mathrm{~Hz}) ; \mathrm{ST} \mathrm{SD}=$ semitone standard deviation; $\mathrm{STR}=$ speaking time ratio; Rate (words per minute); $\mathrm{C}=$ control participants; $\mathrm{A}=$ participants with aphasia; $\mathrm{P}=$ pink noise; $\mathrm{M}=$ =monologue; $\mathrm{PC}=$ phone call; $\mathrm{LC}=$ lively conversation; $\mathrm{CT}=$ cocktail noise; $\mathrm{ES}=\mathrm{effect}$ size; $\mathrm{df}=1,19$ for all measures besides Mean dB and SD dB $(1,18)$. 


\section{Table 8}

ANOVA Results for Between Subject (Control Participants Versus Participants With Aphasia)

Effects on Acoustic Measures

\begin{tabular}{ccccc}
\hline & \multicolumn{4}{c}{ ANOVA Results } \\
\cline { 2 - 5 } Measure & $\mathrm{df}$ & F-ratio & $p$ & Effect Size \\
\hline Mean dB & 1,18 & 3.438 & .080 & .160 \\
SD dB & 1,18 & 0.865 & .365 & .046 \\
Mean F & 1,19 & 0.008 & .928 & $<.001$ \\
ST SD & 1,19 & 0.637 & .435 & .032 \\
STR & 1,19 & 17.544 & $<.001$ & .480 \\
Rate & 1,19 & 17.060 & .001 & .473 \\
\hline
\end{tabular}

Note. Mean $\mathrm{dB}=$ mean intensity $(\mathrm{dB}) ; \mathrm{SD} \mathrm{dB}=$ standard deviation of mean intensity; Mean $\mathrm{F}_{0}$ $(\mathrm{Hz})$; ST SD=semitone standard deviation; $\mathrm{STR}=$ speaking time ratio; Rate (words per minute). 


\section{Figure 3}

Mean and Standard Deviation of Intensity Across Conditions by Group

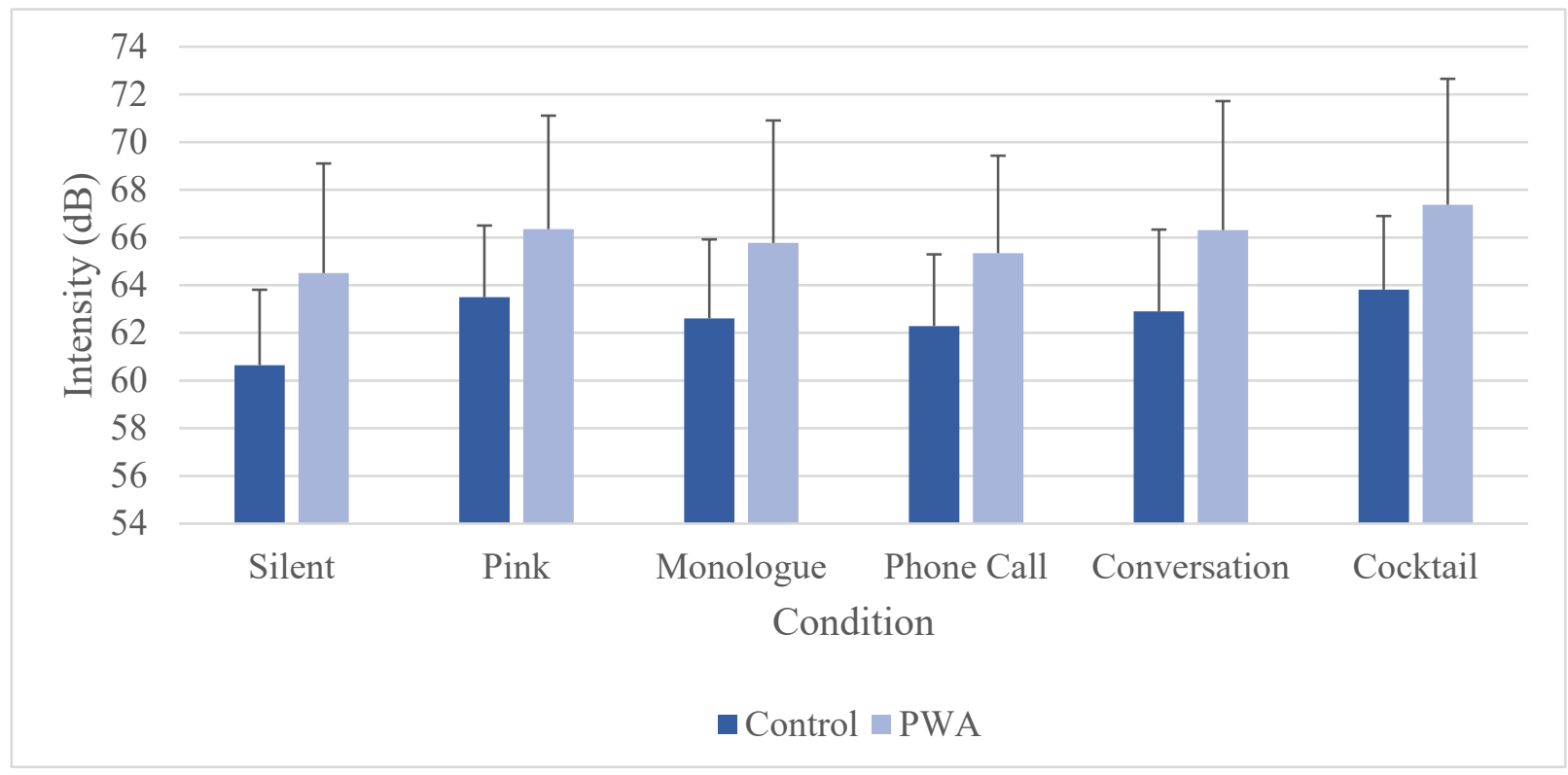

Figure 4

Mean and Standard Deviation of Fundamental Frequency Across Conditions by Sex

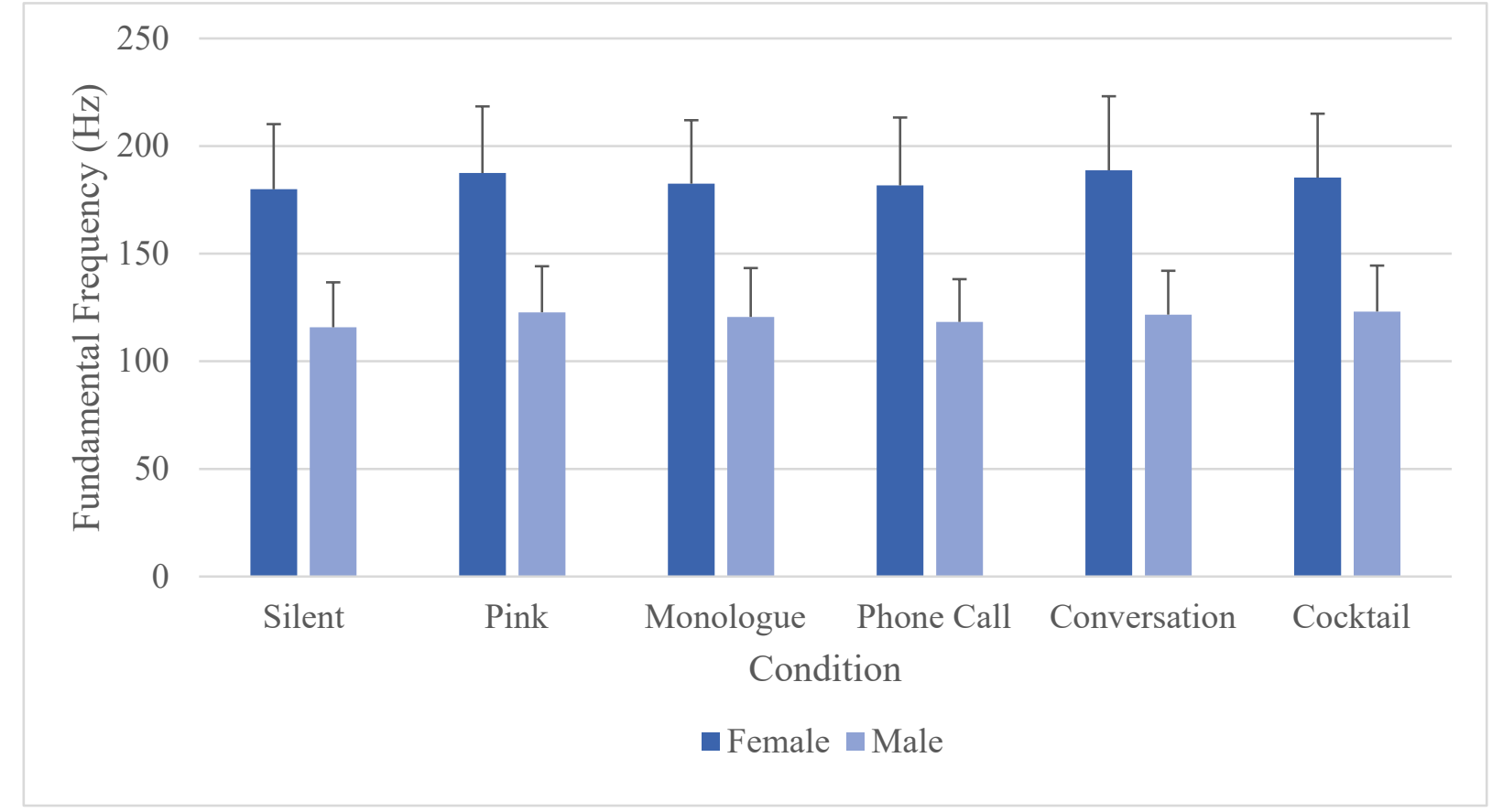




\section{Figure 5}

Mean and Standard Deviation of Speaking Time Ratios by Group

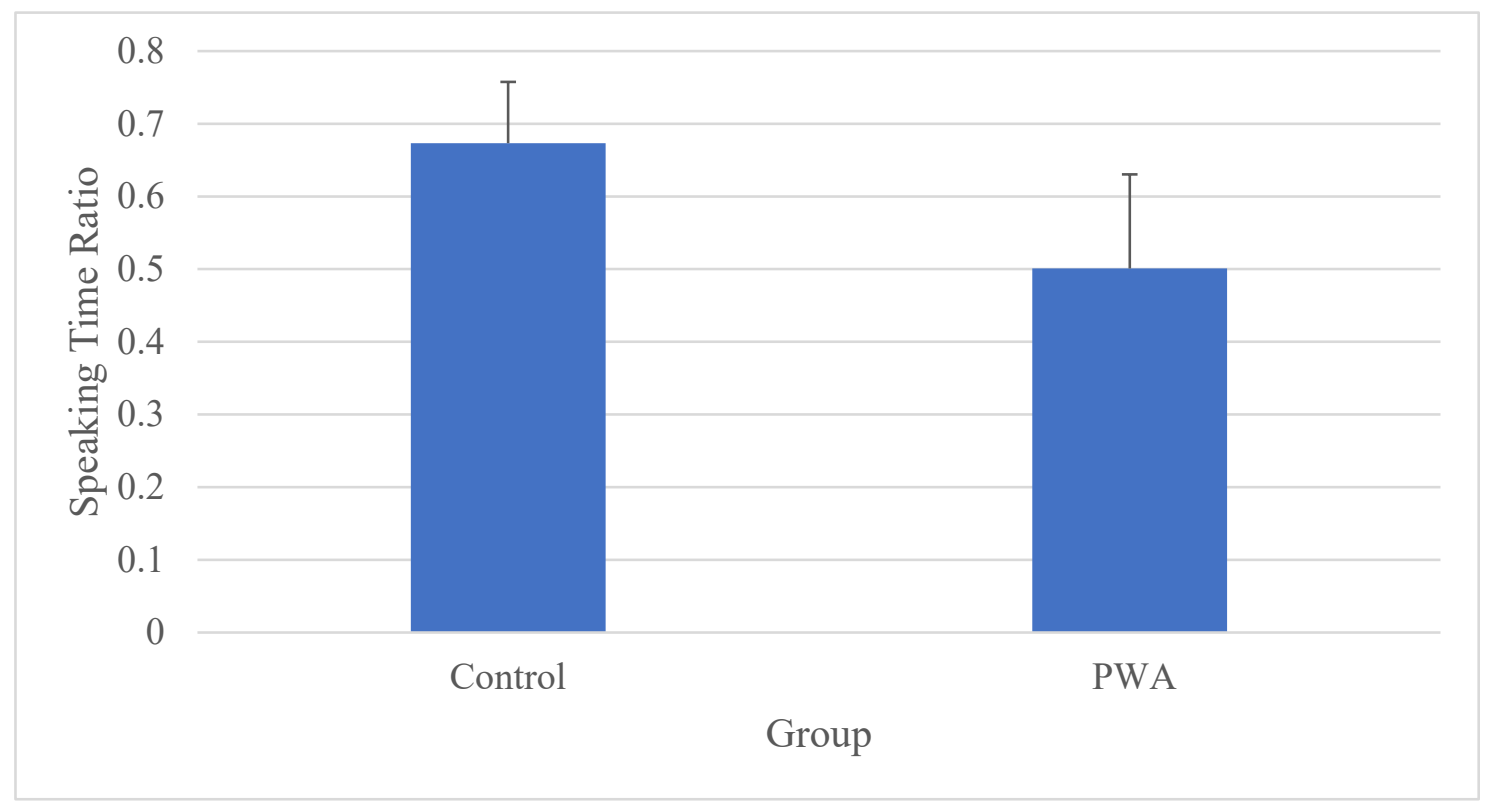

\section{Figure 6}

Mean and Standard Deviation of Speaking Rate by Group

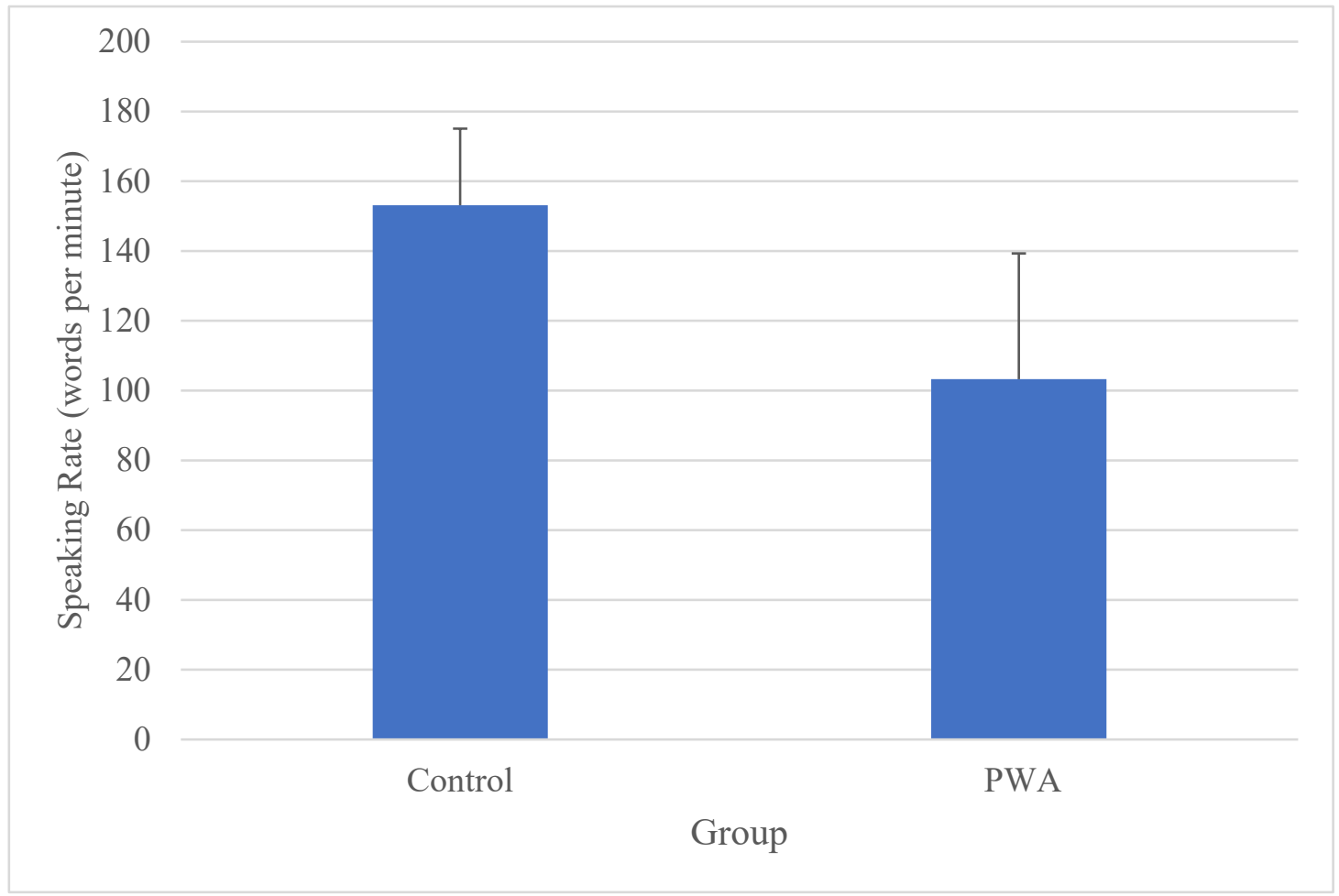




\section{Discussion}

This study investigated how PWA and control participants modify their speech acoustics in the presence of background noise. A further goal of the study was to identify differences in speech acoustics produced in informational versus energetic background noise conditions. Both PWA and control participants increased vocal intensity across background noise conditions. In addition, aside from the monologue and one-sided phone call conditions, both groups of participants demonstrated increases in $\mathrm{F}_{0}$ when speaking in background noise. PWA demonstrated lower speaking time ratios and speaking rates when compared to control participants across conditions. No acoustic changes between speech produced in informational verses energetic background noises were identified.

\section{Intensity}

Previous studies involving speech produced in background noise have reported an increase in vocal intensity (Chapman, 2019; Cooke \& Lu, 2010; Dromey \& Scott, 2016; Hazan \& Baker, 2011; Lu \& Cooke, 2008; Smiljanic \& Gilbert, 2017). This increase is reasoned to occur due to individuals' efforts to make their voices louder (and therefore, more intelligible) than the background noise. Similarly, in the current work, both PWA and control participants spoke more loudly during all background noise conditions than in silence. As all background noise stimuli were perceptually matched to a $60 \mathrm{~dB}$ HL masking noise, a significant increase in intensity across conditions was to be expected.

\section{Fundamental Frequency}

Recent studies have also described rises in $\mathrm{F}_{0}$ during speech produced in noise (Graetzer et al., 2017; Lu \& Cooke, 2009b). Likewise, in the current study, both groups of participants demonstrated significant increases in $\mathrm{F}_{0}$ across three of the five background noise conditions. 
Previous researchers found that as participants increased their vocal intensity, their $\mathrm{F}_{0}$ also rose by approximately half a semitone per $\mathrm{dB}$. This increase was reasoned to occur as the increase in subglottal pressure needed for louder speech can indirectly cause the vocal folds to vibrate faster (Gramming et al., 1988). Both groups of participants in the current work demonstrated louder speech (driven by higher subglottal pressures) across background noise conditions; thus, a passive increase in $\mathrm{F}_{0}$ was also likely to occur.

A significant increase in $\mathrm{F}_{0}$ did not occur with speech produced during the one-sided phone call condition. When creating the phone call stimulus, one speaker's utterances were removed in an effort to simulate a common background noise (i.e., hearing one side of a phone conversation). However, this removal resulted in half of the phone call condition being silent. We reasoned that a significant increase in Fo did not occur during the one-sided phone call condition due the fact that half of the time it was equivalent to the silent baseline condition. We are unsure why mean $\mathrm{F}_{0}$ did not significantly increase in the monologue condition.

\section{Between Group Acoustic Differences}

Previous studies found that the spoken language of PWA changed when faced with attentional demands (Harmon et al., 2019; Murray, 2012; Murray et al., 1998). Specifically, PWA (even mild aphasia) significantly decreased speech rate when compared to a control group while retelling narratives in a dual task condition (Harmon et al., 2019). We hypothesized that due to potential selective attention weaknesses, the PWA would demonstrate greater acoustic changes during the experimental task than the control participants (Murray, 2012). The PWA did have significantly lower speaking time ratios and speaking rates across conditions when compared to the control participants. The researchers anticipated these differences because aphasia is a language disorder that affects individuals' abilities to retrieve words, thereby 
lowering speech rate. Additionally, PWA demonstrated an insignificantly higher intensity across conditions when compared to the control group. This difference might be due to a more effortful speech pattern on the part of the PWA. A previous study found that when 20 young women spoke in two tasks of varying cognitive difficulty, they produced fewer syllables per breath in the more cognitively demanding speaking task. In addition, the women demonstrated increases in lung volume per syllable when required to speak in the more difficult task (Mitchell \& Hoit, 1996). Deficits in selective attention (Murray, 2012) may have increased the cognitive difficulty of the experimental task for PWA. In addition, due to a slower speaking rate PWA may have produced fewer syllables per breath, and potentially, a higher lung volume per syllable. Similar differences in lung volume may explain why PWA had a trend toward higher intensity while retelling the stories when compared to the control group.

\section{Informational Verses Energetic Background Conditions}

The results of recent studies found that informational background noise was more cognitively demanding than energetic background noise (Lidestam et al., 2014; Perham et al., 2016). Additionally, previous research suggests that speakers take advantage of pauses in informational background noise to increase their verbal output, thereby changing the temporal structure of their speech (Cooke \& Lu, 2010). In contrast, there were no significant changes in speaking time ratios and speaking rates as PWA and control participants retold stories in the informational background noises (i.e., the monologue, one-sided phone call, and lively conversation) versus the energetic background noises (i.e., the pink noise and cocktail speech). It is possible that our acoustic analyses did not detect smaller production differences in speech between the two types of background noises. For instance, word segments (i.e., phonemes) could have been produced differently in informational verses energetic conditions. Our speaking rate 
measure using words per minute would not have been able to detect these subtle changes. Also, while efforts were made to simulate common background noises in the experimental stimuli, it is possible that the experimental stimuli were not as disruptive as every day background noises (e.g., a nearby conversation at a social gathering). As a result, the participants may have been able to tune out the informational background noises during the experiment whereas they might have difficulty doing so during everyday interactions. Lastly, as discussed previously, one of the informational background conditions, the one-sided telephone call, was silent approximately half of the time. This silence potentially could have decreased distraction, thereby lessening the effects of informational background noise that previous studies have reported.

\section{Limitations of the Current Work}

We aimed to have at least 15 PWA and 15 control participants take part in the study. Due to difficulty recruiting participants during COVID-19, only 12 PWA originally agreed to participate. As mentioned previously, one of the PWA did not complete the entire experimental task. In addition, one of the potential PWA misinterpreted what aphasia was, and did not actually have an aphasia diagnosis. As a result, the data from only 10 PWA and 11 control participants were included in the statistical analyses, thereby limiting statistical power.

A recent study found that background noises sharing linguistic similarities with experimental tasks increased distractibility (Perham et al., 2016). While efforts were made to simulate everyday background noises in the experimental stimuli, the experimenters did not include background noises that contained similar linguistic content to the content of narrative stories (e.g., having the participants listen to an audio book about a fire while retelling a story about a fire). While individuals may not always hear informational background noises that are pertinent to their own interactions in everyday communication situations, dissimilarities between 
the informational background noises and the content of the narrative stories may have decreased the disruptive potential of the informational background noise stimuli.

Lastly, in an effort to maintain all hesitations in the speech samples, filler words (such as "um") were not manually removed. While the decision to keep filler words may have resulted in more accurate speaking time ratio calculations, it potentially could have inflated speaking rate measures because filler words were counted in the words per minute totals. Inflated speaking rates for speech samples produced in informational background noises could potentially have lessened statistical significance for acoustic differences between speech produced in informational and energetic background noises.

\section{Implications for Future Research}

Future studies investigating the effects of background noise on the speech acoustics of PWA should include more PWA and control participants to increase statistical power. Additionally, it is suggested that researchers consider the amount of silence present in background noise stimuli to ensure that experimental background conditions are sufficiently different from the silent baseline condition. It could also be beneficial for future experimenters to take into consideration whether or not informational background noise conditions that share similar linguistic topics with experimental tasks elicit more significant changes in spoken language (Perham et al., 2016). The results from the questionnaires and interviews may also help in selecting more distracting stimuli for future studies. Another recommendation is to add analyses to future studies that examines the rate of speech segments, as well as proportion of filler words versus intentional utterances during each background noise condition. 


\section{Implications for Practitioners}

As a result of significantly lower speaking time ratios and speaking rates, PWA may take longer participating in social interactions with background noise. A vital component of aphasia treatment is preparing PWA and their communication partners for what they may encounter in different communication contexts. Speech language pathologists can "[provide] education and counseling to significant others to improve attitudes and reduce stigma associated with communication problems" (Hallowell, 2017, p. 442). For instance, clinicians could first teach the communication partners that PWA may have a slower speaking rate, especially in noisy environments. Role playing could then help communication partners practice giving PWA sufficient time to take turns, refraining from interrupting or filling in words, and also removing themselves from noisy environments where possible. PWA could rehearse disclosing their communication disorder to others (e.g., "I have aphasia. It makes it hard for me talk, especially when it is noisy. Please be patient as I express myself').

Although the results did not demonstrate significant acoustic differences between speech produced in informational versus energetic background noise conditions, qualitative research has reported that PWA find informational background noises especially distracting (Baylor et al., 2011). Due to individual differences among PWA, at the start of an intervention period it is recommended that speech language pathologists work with PWA to identify which background noises they find most distracting. Both PWA and their frequent communication partners could be given logs to record background noises they hear frequently, as well background noises that make participating in interactions especially difficult or stressful. In addition, audio clips of both informational and energetic background noises could be played in sessions. PWA could rate perceived level of distractibility of each background noise, while speech-language pathologists 
could take data regarding speech rate, level of fluency, etc. while communicating with the PWA in the various background noises. As mentioned previously, implementing background noises in therapy sessions could assist PWA in developing communication strategies to express themselves more successfully, even in noisy environments.

\section{Conclusion}

This study examined how PWA and control participants changed their speech acoustics while retelling stories in informational and energetic background noises. The results of this study reveal that in the presence of informational and energetic background noises, both PWA and control participants increased their intensity in an effort for their speech to be heard over the background noise. This increase in intensity also resulted in passive rises in mean $\mathrm{F}_{0}$ across three of the five background conditions. PWA demonstrated significantly lower speaking time ratios and speaking rates across conditions when compared to the control group. Further research is recommended to investigate more subtle acoustic changes in speech produced in informational verses energetic background noise conditions. 


\section{References}

Adobe Inc. (2021). Adobe audition (Version 14.0) [Computer program]. Retrieved 01 March 2021 from http://adobe.com/audition

Audacity Team. (2020). Audacity(R): Free Audio Editor and Recorder (Version 2.4.1) [Computer program]. Retrieved 01 June 2020 from https://audacityteam.org/

Baylor, C., Burns, M., Eadie, T., Britton, D., \& Yorkston K. (2011). A qualitative study of interference with communicative participation across communication disorders in adults. American Journal of Speech-Language Pathology, 20(4), 269-287. https://doi.org/10.1044/1058-0360(2011/10-0084)

Boersma, P. \& Weenink, D. (2021). Praat: Doing phonetics by computer (Version 6.1.40) [Computer program]. Retrieved 27 February 2021 from http://www.praat.org/

Chapman, K. N. (2019). The effects of distracting background audio on spontaneous speech [Master's thesis, Brigham Young University]. BYU ScholarsArchive. https://scholarsarchive.byu.edu/etd/7415

Cooke, M., \& Lu, Y. (2010). Spectral and temporal changes to speech produced in the presence of energetic and informational maskers. Journal of the Acoustical Society of America, 128(4), 2059-2069. https://doi.org/10.1121/1.3478775

Di Blasio, S., Shtrepi, L., Puglisi, G., \& Astolfi, A. (2019). A cross-sectional survey on the impact of irrelevant speech noise on annoyance, mental health and well-being, performance and occupants' behavior in shared and open-plan offices. International Journal of Environmental Research and Public Health, 16(2), 280. https://doi.org/10.3390/ijerph16020280 
Dromey, C., \& Benson, A. (2003). Effects of concurrent motor, linguistic, or cognitive tasks on speech motor performance. Journal of Speech, Language, and Hearing Research, 46(5), 1234-1246. https://doi.org/10.1044/1092-4388(2003/096)

Dromey, C., \& Scott, S. (2016). The effects of noise on speech movements in young, middleaged, and older adults. Speech, Language, and Hearing, 19(3), 131-139. https://doi.org/10.1080/2050571X.2015.1133757

Graetzer, S., Bottalico, P., \& Hunter, E. J. (2017). Speech produced in noise: Relationship between listening difficulty and acoustic and durational parameters. Journal of the Acoustical Society of America, 142(2), 974-983. https://doi.org/10.1121/1.4997906

Gramming, P., Sundberg, J., Ternstrom, S., Leanderson, R., \& Perkins, J. (1988). Relationship between changes in voice pitch and loudness. Journal of Voice, 2(2), 118-126. https://doi.org/10.1016/S0892-1997(88)80067-5

Hallowell, B. (2017). Aphasia and other acquired neurogenic language disorders: A guide for clinical excellence. Plural Publishing.

Harmon, T. G. (2020). Everyday communication challenges in aphasia: descriptions of experiences and coping strategies. Aphasiology, 34(10), 1270-1290. https://doi.org/10.1080/02687038.2020.1752906

Harmon, T. G., Jacks, A., Haley, K. L., \& Bailliard, A. (2019). Dual-task effects on story retell for participants with moderate, mild, or no aphasia: Quantitative and qualitative findings. Journal of Speech, Language, and Hearing Research, 62(6), 1890-1906.

https://doi.org/10.1044/2019_JSLHR-L-18-0399 
Hazan, V., \& Baker, R. (2011). Acoustic-phonetic characteristics of speech produced with communicative intent to counter adverse listening conditions. The Journal of the Acoustical Society of America, 130(4), 2139-2152. https://doi.org/10.1121/1.3623753

Johnson, A. F. \& Jacobsen, B. H. (2017). Medical speech-language pathology: A practitioner's guide. Thieme Publishers.

Jones, W. J., Williams, L. S., \& Meschia, J. F. (2001). Validating the questionnaire for verifying stroke-free status (QVSFS) by neurological history and examination. American Heart Association Journal, 32(10), 2232-2236. https://doi.org/10.1161/hs1001.096191

Lidestam, B., Holgersson, J., \& Moradi, S. (2014). Comparison of informational vs. energetic masking effects on speechreading performance. Frontiers in Psychology, 5, 639. https://doi.org/10.3389/fpsyg.2014.00639

Lu, Y., \& Cooke, M. (2008). Speech production modifications produced by competing talkers, babble, and stationary noise. The Journal of the Acoustical Society of America, 124(5), 3261-3275. https://doi.org/10.1121/1.2990705

Lu, Y., \& Cooke, M. (2009a). The contribution of changes in $\mathrm{F}_{0}$ and spectral tilt to increased intelligibility of speech produced in noise. Speech Communication, 51(12), 1253-1262. https://doi.org/10.1016/j.specom.2009.07.002

Lu, Y., \& Cooke, M. (2009b). Speech production modifications produced in the presence of lowpass and high-pass filtered noise. Journal of the Acoustical Society of America, 126(3), 1495-1499. https://doi.org/10.1121/1.3179668

Mitchell, H. L., \& Hoit, J. D. (1996). Cognitive-linguistic demands and speech breathing. Journal of Speech \& Hearing Research, 39(1), 93-104. https://doi.org/10.1044/jshr.3901.93 
Murray, L. L. (2012). Attention and other cognitive deficits in aphasia: Presence and relation to language and communication measures. American Journal of Speech-Language Pathology, 21(2), 51-64. https://doi.org/10.1044/1058-0360(2012/11-0067)

Murray, L. L., Holland, A. L., \& Beeson, P. M. (1998). Spoken language of individuals with mild fluent aphasia under focused and divided-attention conditions. Journal of Speech, Language, and Hearing Research, 41(1), 213-227. https://doi.org/10.1044/jslhr.4101.213

Parasuraman, R. (1998). The attentive brain. Cambridge: MIT Press.

Perham, N., Marsh, J. E., Clarkson, M., Lawrence, R., \& Sörqvist, P. (2016). Distraction of mental arithmetic by background speech. Experimental Psychology, 63(3), 141-149. https://doi.org/10.1027/1618-3169/a000314

Renz, T., Leistner, P., \& Liebl, A. (2018). Auditory distraction by speech: Comparison of fluctuating and steady speech-like masking sounds. Journal of the Acoustical Society of America, 144(2), EL83. https://doi.org/10.1121/1.5048637

Smiljanic, R., \& Gilbert, R. C. (2017). Acoustics of clear and noise-adapted speech in children, young, and older adults. Journal of Speech, Language, and Hearing Research, 60(11), 3081-3096. https://doi.org/10.1044/2017_JSLHR-S-16-0130

Summers, W. V., Pisoni, D. B., Bernacki, R. H., Pedlow, R. I., \& Stokes, M. A. (1988). Effects of noise on speech production: Acoustic and perceptual analyses. Journal of the Acoustical Society of America, 84(3), 917-928. https://www.ncbi.nlm.nih.gov/pubmed/3183209

Wilson, S. M., Eriksson, D. K., Schneck, S. M., \& Lucanie, J. M. (2018). A quick aphasia battery for efficient, reliable, and multidimensional assessment of language function. PLoS ONE, 13(6), Article e0199469. https://doi.org/10.1371/journal.pone.0192773 


\section{APPENDIX A}

\section{Annotated Bibliography}

Baylor, C., Burns, M., Eadie, T., Britton, D., \& Yorkston K. (2011). A qualitative study of interference with communicative participation across communication disorders in adults. American Journal of Speech-Language Pathology, 20(4), 269-287. https://doi.org/10.1044/1058-0360(2011/10-0084)

Objective: The purpose of this study was to investigate the content of the Communicative Participation Item Bank (CPIB, a questionnaire that evaluates the extent to which individuals with communication disorders participate in everyday interactions). The examiners performed this study in order to determine which revisions could be made to improve the content of the CPIB.

Methods: Qualitative data were gathered as 44 individuals with communication disorders answered questions from the CPIB in an interview format. All data were input into Atlas.ti qualitative software, which then coded the data and detected themes within the responses.

Results: One theme that emerged from the CPIB results was that participants' ability to participate in interactions depended in part upon their surroundings. One participant (61-year-old male with Parkinson's disease) reported that background noise was the largest deterrent in participating in conversations. Another participant (67-yearold male with aphasia) reported that informational background noise made it especially difficult to produce coherent spoken language. 
Conclusions: Social interactions can be shaped by individuals' surroundings. Individuals with communication disorders are especially influenced by their communication environments.

Relevance to the Current Study: Twelve of the 44 participants developed communication disorders after strokes. Half of participants in the current study will also have aphasia. In addition, the qualitative data from this study support the current work's hypotheses that 1) background noise does affect speech production, 2) informational masking may be more distracting than energetic masking, and 3) PWA may demonstrate greater changes in speech production than control participants.

Chapman, K. N. (2019). The effects of distracting background audio on spontaneous speech [Master's thesis, Brigham Young University]. BYU ScholarsArchive. https://scholarsarchive.byu.edu/etd/7415

Objective: The purpose of this study was to assess the changes in speech production that occur in different types of noise conditions (specifically, energetic and informational masking).

Methods: Twenty male and 20 female participants answered open-ended questions while listening to six background noise conditions (silent baseline, pink noise, movie dialogue, two speakers debating, classical music, and contemporary music) through closed headphones. After answering the questions, the participants rated which background noise conditions they found most distracting. Mean $\mathrm{F}_{0}, \mathrm{~F}_{0}$ standard deviation in semitones, mean intensity, intensity standard deviation, speaking time ratio, and a subjective rating of fluency were collected. An ANOVA was performed to test for 
statistically significant differences between the background noise conditions and the silent baseline condition.

Results: There was a significant increase in $\mathrm{F}_{0}$ in the speech samples collected during the noise conditions vs. the data collected during the silent baseline condition. There was also a significant increase in mean intensity aside from the pink noise condition. Speaking time ratio increased for all noise conditions except for the classical music condition. Disfluencies increased for all noise conditions aside from the pink noise and classical music conditions. Disfluencies occurred at a higher rate for the debate and contemporary music conditions, which were generally rated as the most distracting conditions by the participants.

Conclusions: The results of this study suggest that speech modifications (such as an increase in $\mathrm{F}_{0}$ and intensity) occur in the presence of noise. In addition, the results imply that disfluencies occur more frequently when participants find background noise conditions more distracting.

Relevance to the current study: This is a foundational study for the current study. In particular, the results from this study have aided the current researchers in selecting appropriate background noise conditions and in deciding to use open back, rather than closed headphones.

Cooke, M., \& Lu, Y. (2010). Spectral and temporal changes to speech produced in the presence of energetic and informational maskers. Journal of the Acoustical Society of America, 128(4), 2059-2069. https://doi.org/10.1121/1.3478775

Objective: The purpose of this study was to examine the effect of energetic and informational masking on interactional vs. non-interactional tasks. The study had two 
specific objectives: 1) determine if speakers take advantage of the temporal/spectral dips that occur in informational maskers when communicating with others, and 2) determine if speakers modify their speech at greater levels when interacting with others.

Methods: Eight participants (four males and four females) were divided into four, same-gender pairs. Each participant worked on Sudoku puzzles for 10-minute increments in eight conditions (quiet, competing speech, speech-shaped noise, and speech-modulated noise with and without their partner). Participants were encouraged to talk through the Sudoku puzzle process either by themselves or with their partners. Responses were transcribed, and word duration, root-mean-square energy, mean $\mathrm{F}_{0}$, spectral distribution, and degree of temporal overlap were recorded. A two-way ANOVA was performed to detect statistically significant differences between the background noise conditions and silence, as well as between communicative vs. non-communicative tasks.

Results: All data gathered in the background noise conditions showed an increase in root-mean-square energy and mean $\mathrm{F}_{0}$ when compared to data gathered in the silent condition. Communicative tasks had a larger increase in mean $\mathrm{F}_{0}$, intensity, and spectral balance, and a decrease in word duration. The degree of temporal overlap was smaller during communicative tasks.

Conclusions: Individuals modify their speech when presented with noise. The results of this study also suggest that when communicating with others, speakers increase speech modifications and actively listen to masking conditions to try to take advantage of the temporal breaks.

Relevance to the current work: Although the current study does not involve communicative tasks as the stimuli are presented, it could be beneficial for the current 
researchers to use degree of temporal overlap measures to see if the test participants take advantage of temporal breaks while retelling the narrative stories in informational masking conditions.

Di Blasio, S., Shtrepi, L., Puglisi, G. E., \& Astolfi, A. (2019). A cross-sectional survey on the impact of irrelevant speech noise on annoyance, mental health, and well-being, performance and occupants' behavior in shared and open-plan offices. International Journal of Environmental Research and Public Health, 16(2), 280. https://doi.org/10.3390/ijerph16020280 Objective: This study had three objectives: 1) determine how irrelevant speech noise (IRS) affects work performance/mental health, 2) examine if correlations between personal traits (e.g., gender) and noise annoyance levels exist, and 3) explore whether or not employees would be willing to use an in-office system to monitor IRS.

Methods: Employees from 19 companies, five research facilities, and one university were recruited and asked to fill out a questionnaire via email. 1,078 employees filled out the survey, which contained a summary outlining the purpose of the survey, as well as 17 questions on background information and opinions regarding IRS. Statistical analyses (Mann-Whitney U test and Kruskal-Wallis test) were applied to the questionnaire results to determine if statistically significant correlations existed between the responses on the background information section and the responses on the opinions regarding IRS section.

Results: The results from the statistical analyses suggested that the employees working in open office spaces (rather than smaller, shared office spaces) were more 
annoyed by IRS and had a more difficult time working due to IRS. In addition, the results suggested that women are more negatively impacted by IRS than men.

Conclusions: This study suggests that women working in open office spaces are the most likely subset of employees to experience negative work impacts due to IRS.

Relevance to the current work: Three of the background conditions in the current study (the lively conversation, the monologue, and the one-sided telephone call) could be considered IRS, for they are noisy and intelligible. In addition to knowing that these background noise conditions increase the cognitive load during the narrative retell task, it is beneficial to know other potential side effects of these background noise conditions.

Dromey, C., \& Benson, A. (2003). Effects of concurrent motor, linguistic, or cognitive tasks on speech motor performance. Journal of Speech, Language, and Hearing Research, 46(5), 1234-1246. https://doi.org/10.1044/1092-4388(2003/096)

Objective: The purpose of this study was to assess how different tasks (specifically motor, linguistic, and cognitive tasks) affect lip/jaw movements.

Methods: Ten male and 10 female participants repeated the phrase, "Mr. Piper and Bobby would probably pick apples," 15 times during six trials. Three of the trials involved solely repeating the phrase, while the other three trials involved repeating the phrase while simultaneously completing motor (placing nuts, bolts, and washers together), linguistic (generating verbs), and cognitive (counting backwards) tasks. Lip and jaw movements were transduced using strain gauge instrumentation, and duration, displacement/velocity, correlation of upper/lower lip, and spatiotemporal index (a measure used to show whether or not movements between the repetitions were consistent) were recorded. An ANOVA was used to determine if statistically significant 
differences were present between data collected during the speech-only condition and the other three conditions.

Results: During the motor task participants had decreased lip velocity/displacement. In both the cognitive and linguistic distractor conditions, the spatiotemporal index for the lower lip increased (i.e., became less consistent), especially for the male participants. Interestingly, during these two conditions, there was also strong negative correlation between the upper/lower lip, suggesting normal lip movements. Utterance duration significantly decreased during the cognitive task.

Conclusions: Concurrent, distracting tasks influence speech production (e.g., the results from the motor task suggest that articulatory undershoot may occur while dividing attention between speaking/other motor movements). During cognitive/linguistic tasks, speakers may rely on strongly coordinated lip movements in order to devote more attention to the distracting task.

Relevance to the current work: In the current work, acoustic measures will be recorded during different background noise conditions. The results from this study suggest that as the current researchers present distracting stimuli to the test participants, their speech movements, and therefore acoustic measures, will change.

Dromey, C., \& Scott, S. (2016). The effects of noise on speech movements in young, middleaged, and older adults. Journal of Speech, Language, and Hearing, 19(3), 131-139. https://doi.org/10.1080/2050571X.2015.1133757

Objective: The purpose of this study was to assess how different types of background noise conditions influence changes in articulatory movements across the lifespan. Specifically, this study sought to determine how conditions involving spoken 
language (i.e., informational masking) influenced movements when compared to conditions involving steady noise (i.e., energetic masking).

Methods: Thirty male and 30 female subjects participated in the study, with their ages ranging from 20-70 years. The participants read the phrase, "In Panama, most people prefer to travel by bus, bike, or boat," 15 times in the five different background noise conditions (one person reading a novel, two readers, six readers, pink noise, and a silent condition). During the repetitions, lip/jaw movements were measured using strain gauge instrumentation. Phrase duration, displacement/velocity of the lower lip, upper/lower lip correlation, velocity peaks (with fewer peaks representing more stable movements), spatiotemporal index of the lower lip, and intensity were recorded. An ANOVA was used to determine if significant differences were present between the speech movements/acoustics associated with the four masking conditions and the silent baseline condition.

Results: Repetitions produced with the single-reader condition had shorter phrase durations than the silent condition. The lower lip velocity increased, the intensity increased, and the number of velocity peaks decreased across all masking conditions when compared to the baseline. The spatiotemporal index was lower for the pink noise/six-reader condition when compared to the silent condition.

Conclusions: As the Lombard effect suggests, speech produced in noise is often associated with unintentional increases in SPL. Additionally, the results from this study suggest that louder speech may make articulatory gestures smoother.

Relevance to the current work: In this study, no significant results were found between speech production in informational masking vs. energetic masking conditions. 
The results from this study are aiding the current researchers in creating a study that will hopefully maximize the differences between informational masking vs. energetic masking (e.g., different background noise conditions and narrative retell tasks, rather than repetition tasks).

Gramming, P., Sundberg, J., Ternstrom, S., Leanderson, R., \& Perkins, J. (1988). Relationship between changes in voice pitch and loudness. Journal of Voice, 2(2), 118-126. https://doi.org/10.1016/S0892-1997(88)80067-5

Objective: The purpose of this study was to investigate the relationship between $\mathrm{F}_{0}$ and intensity.

Methods: Nine male singers and nine male non-singers participated in the study. Additionally, 10 male and 10 female voice patients took part in the study. Participants were instructed to read text passages under varying conditions. The voice patients read the passages at a normal voice volume in silence and while listening to masking noise. The singers and non-singers were instructed to read passages in silence and in masking noise at a normal voice volume, at a volume loud enough for an audience to hear, and at the loudest volume possible. Also, the singers and non-singers sang triads or performed pitch glides at the lowest and loudest volumes possible to create voice range profiles. Statistical analyses were performed to identify correlations between $\mathrm{F}_{0}$ and vocal intensity in the recorded samples. Changes in $\mathrm{F}_{0}$ among the different sound conditions were recorded as semitones since each participant had a different baseline $\mathrm{F}_{0}$.

Results: Mean $\mathrm{F}_{0}$ and vocal intensity were strongly correlated, with mean $\mathrm{F}_{0}$ increasing by about half a semitone per $\mathrm{dB}$. Additionally, the participants who were singers changed their $\mathrm{F}_{0}$ more significantly than other groups of participants. 
Conclusions: $\mathrm{F}_{0}$ increases as intensity increases. The researchers reasoned that changes in $F_{0}$ occur due to the variations in subglottal pressure needed to create different voice loudness levels.

Relevance to the current work: Both mean intensity and mean $\mathrm{F}_{0}$ will be recorded and analyzed throughout the study. Thus, it is beneficial to understand the relationship between these two acoustic measures.

Graetzer, S., Bottalico, P., \& Hunter, E. J. (2017). Speech produced in noise: Relationship between listening difficulty and acoustic durational parameters. Journal of the Acoustical Society of America, 142(2), 974-983. https://doi.org/10.1121/1.4997906

Objective: This study had three objectives related to listening difficulty (LD), or the percentage of words that are difficult to hear: 1) examine the LD of speech in different noise/style backgrounds, 2) analyze the durational/spectral changes in the speech samples, and 3) determine if these durational/spectral changes could predict LD. Methods: Nineteen participants read the "Rainbow passage" in normal and loud voices under two conditions (40.5 dB(A) (the intensity of the ventilation system) and babble noise at $61 \mathrm{~dB}(\mathrm{~A}))$. The amplitudes of the speech samples were standardized, and they were mixed with pink noise to create a signal-to-noise ratio of $-6 \mathrm{~dB}$. 20 listeners rated the $\mathrm{LD}$ of the speech samples, and vowel length, $\mathrm{F}_{0}$, and spectrum balance were measured.

Results: Speech produced during the louder babble background noise was associated with a raised $\mathrm{F}_{0}$, a more balanced spectrum (i.e., a. spectrum with more energy located in frequencies between 1 and $4 \mathrm{kHz}$ ), and longer vowels. The amount of spectral energy was the only variable that predicted the LD for the 20 listeners. 
Conclusions: Similar to the formant tuning that occurs when singers increase frequency energy at $3 \mathrm{kHz}$ to be heard over orchestras, speakers producing speech in noisy conditions shift frequency energies to minimize the effects of energetic masking.

Relevance to the current work: The results from this study suggest that spectrum balance plays a large role in whether or not a listener has difficulty listening to a speech sample. Thus, it may be beneficial to measure spectrum balance in the current work.

Hallowell, B. (2017). Aphasia and other acquired neurogenic language disorders: A guide for clinical excellence. Plural Publishing.

Relevance to the current work: This textbook clearly outlines what aphasia is, as well as what aphasia is not. Specifically, this textbook teaches that aphasia is acquired (i.e., a person is not born with aphasia), that aphasia has neurological roots (i.e., aphasia occurs due to damage in Broca's or Wernicke's areas), that aphasia can impact both language production and comprehension in various aspects of life (e.g., speaking, reading, and writing), and that aphasia is considered a language disorder (i.e., it does not affect an individual's intellect) (p. 4).

Harmon, T.G. (2020) Everyday communication challenges in aphasia: Descriptions of experiences and coping strategies. Aphasiology, 34(10), 1270-1290. https://doi.org/10.1080/02687038.2020.1752906

Objective: The purpose of this study was to learn more about the everyday communication difficulties people with aphasia (PWA) encounter, as well as how PWA cope with these difficulties.

Methods: Twenty-one individuals with mild to moderate aphasia participated in this study, which consisted of semi-structured interviews that were held immediately after 
an experiment for another study. In the other study, the PWA retold short narrative stories to a responsive communication partner without distractions, to a responsive communication partner with distractions in the form of beeps, and to a nonresponsive communication partner. The intent of performing the interviews immediately after this narrative study was to help prime the PWA so they could easily share difficult communication experiences with the interviewer. The interviews were transcribed and then subsequently coded into three categories: relationships, environmental distractions, and coping.

Results: Six PWA mentioned in their interviews that they found background noise to be especially distracting while communicating. Multiple PWA shared that when they encounter noisy backgrounds, they refrain from participating in conversations with others. In terms of coping with distracting background noise, PWA reported closing their eyes in order to focus on one thing at a time and ensuring that they did not multi-task while conversing in noisy background.

Conclusions: Background noise increases communication difficulties for PWA. Currently, there are very few clinical practices that aid PWA in interacting with others in noisy backgrounds.

Relevance to the current work: In the current study, PWA will retell narrative stories in six background noise conditions. The researchers hope to determine which conditions are most distracting for PWA. This is so that clinicians can incorporate these distracting conditions into intervention sessions to prepare PWA for the noise they will encounter outside of the clinic. 
Harmon, T. G., Jacks, A., Haley, K. L., \& Bailliard, A. (2019). Dual-task effects on story retell for participants with moderate, mild, or no aphasia: Quantitative and qualitative findings. Journal of Speech, Language, and Hearing Research, 62(6), 1890-1906. https://doi.org/10.1044/2019_JSLHR-L-18-0399

Objective: This study had two purposes: 1) investigate how performing tasks while retelling short stories affected the accuracy and speed of spoken language in PWA, and 2) evaluate individuals' emotional responses after performing narrative retell tasks.

Methods: Thirty-three participants took part in this study (21 PWA, 12 control participants). Each participant retold short stories in two conditions. First, in silence, and then while listening to high and low tones. During the second retell, participants were also instructed to differentiate between high and low tones. Quantitative data (participants' effort levels, language accuracy, and speed of spoken language) were recorded. After the story retells, participants shared their experiences during semi structured interviews. Interviews were transcribed and then analyzed using Atlas.ti 8.1.3 (a software used to detect themes between qualitative responses).

Results: There was a significant increase in participants' perceived effort levels during the dual task retell. In addition, individuals with mild aphasia reported significantly greater levels of effort than control participants. Language accuracy, as well as speed of spoken language (i.e., rate in words per minute and number/length of pauses) were both significantly lower for PWA. While participants with moderate aphasia did not experience significant changes in speech rate between the two conditions, control participants and participants with mild aphasia experienced significant changes in pauses and speech rate during the dual task condition. For the qualitative study, data analysis 
revealed two themes: 1) participants negatively reacted to the dual task retell, and 2) participants found ways to cope with the challenges associated with the dual task retell. Participants with mild aphasia mentioned using strategies to perform the two tasks simultaneously (e.g., continuing the retell even if mistakes were made), while control participants and participants with moderate aphasia did not.

Conclusions: Performing two tasks at once can influence language accuracy, as well as rate of speech (especially in PWA). When presented with tasks that increase cognitive demands (like the dual task retell), participants may use strategies to increase focus.

Relevance to the current work: Similar to this study, the current work will investigate how dual tasks influence language accuracy, as well as speech acoustics (like speech rate). The qualitative study also supports the rationale behind the current work; if investigators can determine which background noises create the most significant changes in language accuracy and speech acoustics, these background noises can be used in speech therapy sessions to help participants develop communication strategies in background noise.

Hazan, V., \& Baker, R. (2011). Acoustic-phonetic characteristics of speech produced with communicative intent to counter adverse listening conditions. Journal of the Acoustical Society of America, 130(4), 2139-2152. https://doi.org/10.1121/1.3623753 Objective: This study had two objectives: 1) compare the changes in speech production of talkers communicating in a challenging environment, in an ideal environment, and performing speech production tasks in an imaginary challenging 
condition (e.g., being instructed to read with clear articulation), and 2) determine if the changes speakers make are catered to specific challenging situations.

Methods: Forty subjects participated in the study. The subjects were divided into 20, same-gender pairs. During three sessions, the pairs were placed in separate rooms and communicated over a head-set system and found differences in paired images. During one of the sessions, no barriers were present between the communication partners, but during the subsequent sessions, babble masking or spectrum degradation were added to the interaction to create a challenging communicative environment. Additionally, all participants were instructed to read 144 sentences in either a clear or casual manner. $\mathrm{F}_{0}$ median/range, long-term average spectrum, word duration, and median vowel formants were analyzed from all recordings. Six samples from the various tasks were randomly collected from each participant and were rated by 36 listeners. An ANOVA was performed to determine if statistically significant differences were present between data gathered in background noise conditions vs. silence, communicative tasks vs. noncommunicative tasks, and casual vs. clear speech tasks.

Results: In the clear reading condition, speakers had a higher median $\mathrm{F}_{0}$, $\mathrm{F}_{0}$ range, and a decrease in speech rate when compared to the other conditions. In the babble condition, $\mathrm{F}_{0}$ median/range and intensity increased more when compared to the spectrum degradation condition. Even without an $\mathrm{F}_{0}$ and intensity increase, the spectrum degradation condition was rated as more intelligible than the speech produced without communication barriers.

Conclusions: Because a raising of $\mathrm{F}_{0}$ and intensity would not increase intelligibility in the spectrum degradation condition, the researchers concluded that 
speakers actively tailor their speech to what the listener most needs to hear in difficult communication environments.

Relevance to the current work: Although frequently mentioned in the research, the results from this study indicate that an increase in $\mathrm{F}_{0}$ and intensity do not always increase intelligibility. Thus, in the current work, it will be crucial to measure acoustic variables other than mean $\mathrm{F}_{0} /$ intensity.

Johnson, A. F. \& Jacobsen, B. H. (2017). Medical speech-language pathology: A practitioner's guide. Thieme Publishers.

Relevance to the current work: This textbook provides information on the four main subtypes of cognitive attention (sustained attention, selective attention, divided attention, and alternating attention). This textbook describes that selective attention is crucial in helping individuals perform tasks in the face of distraction, as selective attention aids in filtering out irrelevant stimuli (p. 32). Selective attention relates to the current work because participants will use selective attention to decrease focus on background noise and increase focus on the story retell tasks.

Jones, W. J., Williams, L. S., \& Meschia, J. F. (2001). Validating the questionnaire for verifying stroke-free status (QVSFS) by neurological history and examination. American Heart Association Journal, 32(10), 2232-2236. https://doi.org/10.1161/hs1001.096191

Objective: The purpose of this study was to validate the Questionnaire for Verifying Stroke-Free Status (QVSFS) by comparing QVSFS results to neurological information and medical examinations of a population with a high prevalence of strokes (previously the QVSFS was validated using a population with a low prevalence of strokes). 
Methods: One hundred fifty-five outpatients from Veterans Administration stroke and medicine facilities were administered the QVSFS by a research assistant. A score of zero (meaning no questions were answered "yes") corresponded with stroke-free status. After administrations of the QVSFS, a neurologist performed a medical examination on each outpatient and determined whether or not each patient was stroke-free. Both the research assistant and the neurologist were blind to the patients' stroke statuses.

Results: The probability of accurately classifying a stroke-free patient was $96 \%$, while the probability of accurately determining a patient who had had a stroke was $71 \%$.

Conclusions: The QVSFS accurately identified a high percentage of stroke-free individuals, even in a population with a high prevalence of strokes.

Relevance to the current work: The QVSFS will be administered to control participants prior to the experimental condition to ensure that they have not had strokes, and therefore, aphasia.

Lidestam, B., Holgersson, J., \& Moradi, S. (2014). Comparison of informational vs. energetic masking effects on speechreading performance. Frontiers in Psychology, 5, 1-7. https://doi.org/10.3389/fpsyg.2014.00639 Objective: The purpose of this study was to separate the effects of informational and energetic masking. The researchers sought to determine if reading in the masking conditions was distracting due to attentional problems caused by energetic masking, or phonological processing problems caused by informational masking.

Methods: Twenty-three participants read high-frequency Swedish words in a steady state noise condition, a four-talker babble condition, and in silence. The words were only read visually so that acoustic speech production did not combine the effects of 
energetic and informational masking. The participants were asked to estimate their speech reading accuracy, how much effort it took to read the stimuli in the different conditions, and how distracting each condition was.

Results: Three post-hoc t-tests (in combination with Bonferroni alpha) determined that a significant difference was present between the four-talker babble and silent conditions in terms of speech reading accuracy. Both the four-talker babble and steady state noise conditions were perceived as being equally distracting.

Conclusions: The results suggest that informational masking may be more distracting than energetic masking. This may be because of difficulties with phonological processing; separating the words in the speech reading task from the words in the masking signal may be more cognitively demanding.

Relevance to the current work: A main component of the current study is understanding how different masking conditions (e.g., informational and energetic conditions) affect participants' cognitive loads, especially because half of the participants will have aphasia.

Lu, Y., \& Cooke, M. (2008). Speech production modifications produced by competing talkers, babble, and stationary noise. Journal of the Acoustical Society of America, 124(5), 32613275. https://doi.org/ 10.1121/1.2990705

Objective: The purpose of this study was to determine if the number of talkers speaking $(\mathrm{N})$ influences speech production behaviors, with a small $\mathrm{N}$ coinciding with informational masking, and a large $\mathrm{N}$ corresponding with energetic masking. Methods: Eight participants read 50 Grid sentences (i.e., sentence-like utterances containing six words) in 11 conditions. Babble masking was presented with one, two, 
four, eight, and 16 and infinity talkers (speech-shaped noise) at a level of $80 \mathrm{~dB}$. The onetalker and infinity-talker babbles were also presented at 82 and $96 \mathrm{~dB}$. Baseline was measured with a silent noise condition. The sentences were recorded and phrase duration, RMS energy, mean $\mathrm{F}_{0}$, spectral balance, sentence start time (i.e.., initiation of reading after the start of the masker), number/duration of pauses, and voiced to unvoiced ratio was measured. An ANOVA test was used to determine if statistically significant differences were present between the background noise conditions and the silent noise condition, as well as between the different intensities of the one-talker and infinity-talker babble conditions. 12 listeners then rated the intelligibility of the sentences.

Results: An increase in intensity as well as a raising of mean $\mathrm{F}_{0}$, were statistically significant between the babble conditions and the silent condition. Intelligibility was rated higher for noise-induced speech when compared to speech produced in the silent condition, with intelligibility ratings increasing as number of speakers increased.

Conclusions: Speech modifications produced in noise help speakers increase intelligibility. As the number of speakers and intensity of masking increases (i.e., as the energetic content increases), speech modifications become more evident.

Relevance to the current work: The current work also has a silent condition, two one-talker conditions, a two-talker condition, and a six-talker condition (i.e., cocktail speech). This study is helpful in terms of considering how speech patterns may change in the current study according to how many speakers are talking in each condition.

Lu, Y., \& Cooke, M. (2009a). The contribution of changes in $\mathrm{F}_{0}$ and spectral tilt to increased intelligibility of speech produced in noise. Speech Communication, 51(12), 1253-1262. https://doi.org/10.1016/j.specom.2009.07.002 
Objective: This study had two objectives related to components of Lombard speech: 1) determine the contributions of raising $\mathrm{F}_{0}$ and spectral tilt to overall intelligibility, and 2) determine if there are variables outside of increased $\mathrm{F}_{0}$ and spectral tilt that increase intelligibility.

Methods: Test stimuli was gathered from a study performed by Lu \& Cooke in 2008. Test participants had produced Grid sentences (i.e., a sentence-like utterance containing six words) in a quiet condition and in three speech-shaped noise conditions at 82,89 , and $96 \mathrm{~dB}$. Sentences collected in the quiet conditions were manipulated using computer software to either artificially increase $\mathrm{F}_{0}$, artificially tilt the spectrum to make it more balanced, or manipulate both $\mathrm{F}_{0}$ and spectral tilt. 32 participants then listened to a combination of the original sentences collected in the quiet condition, the three types of manipulated sentences, and the original sentences collected during the speech-shaped noise background conditions. A glimpse area (i.e., area where key acoustic information can be "glimpsed" due to a slower speech rate) was also calculated for the sentences collected during the speech-shaped noise background conditions.

Results: The sentences where $\mathrm{F}_{0}$ was manipulated were not rated as more intelligible when compared to the sentences collected in the quiet condition. The sentences where a spectral shift artificially occurred were rated as more intelligible than the baseline sentences, but were not rated as intelligible as the sentences collected during the speech-shaped noise conditions. Glimpse area calculations demonstrated that glimpse area was higher in noise-induced sentence productions than the other sentences. 
Conclusions: This study suggests that a more balanced spectrum and a higher glimpse area increase overall intelligibility. On the other hand, an increased $\mathrm{F}_{0}$ does not seem to contribute to intelligibility.

Relevance to the current work: In the current work, we will be recording average $\mathrm{F}_{0}$, as well as $\mathrm{F}_{0}$ standard deviation in semitones. Speaking time ratio and speaking rate will also be calculated. The current researchers have not yet planned to calculate glimpse area for the speech samples, but the results from this study reiterate the importance of calculating durational measures since a slower duration provides more time for glimpses of key acoustic information.

Lu, Y., \& Cooke, M. (2009b). Speech production and modifications produced in the presence of low-pass and high-pass filtered noise. Journal of the Acoustical Society of America, 126(3), 1495-1499. https://doi.org/10.1121/1.3179668 Objective: The purpose of this study was to determine whether or not speakers actively shift their spectral energy when listening to different types of masking noise.

Methods: Each of the eight, English speaking participants produced 30 Grid sentences in five background noise conditions (one full-band, two high-pass, and two low-pass filtered conditions). Grid sentences were defined as sentence-like utterances containing six words (e.g., "bin green at K 4 now”). Root mean square energy, average $F_{0}$, average F1, and spectral center of gravity were calculated for each of the samples using Praat. MANOVAs were performed between the acoustic measurements and the five conditions to determine if acoustic changes between conditions were statistically significant. 
Results: The speech produced in the low-pass conditions had a higher mean $\mathrm{F}_{0}$, F1, and spectral center of gravity. The speech produced in the full-band and high-pass filtered conditions showed higher acoustic measurements as well. No statistically significant changes between conditions were shown.

Conclusions: The results from this study suggest that speakers do not actively shift their spectral energy while producing speech in different masking conditions; rather, changes in spectral energy occur in conjunction with other acoustic changes.

Relevance to the current work: Although the spectra of the six background noise conditions used in the current work will differ, the results from this study suggest that increases in the spectral center of gravity might occur as participants produce speech across all conditions.

Mitchell, H. L., \& Hoit, J. D. (1996). Cognitive-linguistic demands and speech breathing. Journal of Speech \& Hearing Research, 39(1), 93-104. https://doi.org/10.1044/jshr.3901.93

Objective: The purpose of this study was to investigate whether or not participating in more cognitively demanding speaking tasks affected speech breathing patterns in participants.

Methods: Twenty young women participated in the study. Participants' breathing patterns were monitored by respiratory magnetometers as they spoke about a topic freely (more cognitively demanding), as well as a topic after preparing an outline (less cognitively demanding). Spoken language was transcribed and compared with recorded lung volumes. 
Results: During the more cognitively demanding tasks, participants produced fewer syllables per breath. In addition, their average lung volume increased per syllable.

Conclusions: More cognitively complex tasks may influence speech breathing patterns. Specifically, more difficult tasks may increase average lung volume per syllable, which may also increase intensity in speech.

Relevance to the current work: In the current work, intensity will be analyzed. Previous studies have found that intensity of speech produced in noise increases due to efforts to increase the signal-to-noise ratio. This study provides an additional suggestion as to why intensity may increase during cognitively demanding tasks.

Murray, L. L. (2012). Attention and other cognitive deficits in aphasia: Presence and relation to language and communication measures. American Journal of Speech-Language Pathology, 21(2), 51-64. https://doi.org/10.1044/1058-0360(2012/11-0067) Objective: This study was performed to investigate the correlation between cognitive deficits (specifically attention) and language performance in individuals with aphasia.

Methods: Thirty-nine individuals with aphasia and 39 control participants were a part of the study. All participants completed a variety of cognitive assessments. To specifically investigate attention, the participants were administered subtests of The Test of Everyday Attention (TEA). One of the administered subtests was the Elevator Counting with Distraction (ECD), which is used to investigate auditory selective attention.

Results: The individuals with aphasia received significantly lower scores on the TEA than the control participants. Specifically, an independent $t$-test demonstrated that 
PWA scored significantly lower on the ECD subtest than the control participants $(t(64.6)=7.032, \mathrm{p}<.0001)$.

Conclusions: Although aphasia has historically been classified as purely a language disorder, cognitive deficits (like attentional deficits) may contribute to PWAs' overall language functioning.

Relevance to the current work: In addition to quantitatively measuring speech and language performance, the results from the experimental condition (retelling stories with background noise) will indirectly give the experimenters information regarding PWAs' attentional skills (specifically selective attention).

Murray, L. L., Holland, A. L., \& Beeson, P. M. (1998). Spoken language of individuals with mild fluent aphasia under focused and divided-attention conditions. Journal of Speech, Language, and Hearing Research, 41(1), 213-227. https://doi.org/10.1044/jslhr.4101.213 Objective: The purpose of this study was to investigate the relationship between attention and production of spoken language in PWA.

Methods: Eight control participants and 14 PWA participated in the study. Participants performed tasks that examined both focused attention and divided attention. First, participants described pictures in silence. The participants then listened to tones and were asked to either discriminate the tones or describe pictures. Lastly, participants were instructed to complete both the tone discrimination and picture description tasks at once. Picture descriptions were divided into utterances. Grammatical, lexical, and pragmatic language components were analyzed using Codes for the Human Analysis of Transcripts (CHAT). 
Results: During all three conditions, PWA performed significantly poorer than control participants in regard to grammatical, lexical, and pragmatic language components. Additionally, while control participants' picture descriptions did not show significant differences when performed in silence vs. during the tone discrimination task, PWAs' descriptions differed significantly. Specifically, their descriptions during the dual task had fewer grammatically accurate utterances and more word-finding difficulties.

Conclusion: Attentional deficits may play a role in PWAs' language deficits, especially during tasks that require divided attention.

Relevance to the current work: Although the current work will investigate how PWAs' spoken language is affected by selective attention tasks, this study suggests that performing tasks while being distracted in any form (like discriminating tones or filtering out background noise) significantly influences PWAs' spoken language.

Parasuraman, R. (1998). The attentive brain. Cambridge: MIT Press.

Relevance to the current work: A main idea of this book suggests that attention is not an entity. Rather, attention interacts with other processes (e.g., the cognitive process of formulating speech and language) (p. 3).

Perham, N., Marsh, J. E., Clarkson, M., Lawrence, R., \& Sörqvist, P. (2016). Distraction of mental arithmetic by background speech. Experimental psychology, 63(3), 141-149. https://doi.org/10.1027/1618-3169/a000314

Objective: The purpose of this study was to determine whether a background noise condition comprised of ascending numbers or descending numbers was more distracting while participants completed addition math problems. Specifically, this study sought to assess whether the response-priming hypothesis (i.e., when the content of 
distracting noise is disruptive because it is similar to the process used to complete the math problems) or the inference-by-content hypothesis (i.e., numbers in general will be disruptive because they overlap with the numbers used in the math problems) was more responsible for the changes in arithmetic performance.

Methods: Thirty-three undergraduate students with typical vision/hearing from a university in Wales participated in the study. The subjects completed 20 addition problems of similar difficulty in 3 background noise conditions (ascending numbers, descending numbers, and silence). An ANOVA test was used to determine if background noise conditions showed statistically significant differences in terms of number of math errors committed.

Results: The analysis showed that there was statistical significance in the difference between type of background noise and number of errors. The undergraduate students committed the most errors in the ascending condition, some errors in the descending condition, and the least amount of errors in the silent condition.

Conclusions: The results from this study suggest that the response-priming hypothesis is a large determinant in the extent of how distracting a relevant background noise condition will be. In this particular study, ascending numbers provided potential answers to the addition problems, making this condition especially distracting.

Relevance to the current work: In the current study, none of the background noise conditions intentionally contain words that could be potential responses for the narrative retell tasks. However, if any of the background noise conditions unintentionally have words that could influence the retell responses, these conditions may be perceived as more distracting. 
Renz, T., Leistner, P., \& Liebl, A. (2018). Auditory distraction by speech: Comparison of fluctuating and steady speech-like masking sounds. Journal of the Acoustical Society of America, 144(2), EL83. https://doi.org/10.1121/1.5048637

Objective: This study had two objectives related to working memory: 1) determine if speech-shaped stationary noise is more efficient in masking distracting speech than stationary noise during a number recall task, and 2) find out if speech samples masked by speech-shaped noise or by stationary noise are perceived as being more annoying during the recall task.

Methods: Twenty-four German-speaking participants completed 144 number recall sequences while listening to 12 background noise conditions (10 mixed conditions that entailed differing amounts of speech-shaped and stationary masking, one silent condition, and one unmasked speech condition). The number of errors committed by participants during the 12 sequences for each background noise condition were marked and averaged. After each noise condition was played, the participants marked their level of annoyance on a scale from $0-10(0=$ not annoying, $10=$ very annoying $)$. ANOVA tests were performed to determine if number of errors and level of annoyance significantly differed between the background noise conditions.

Results: The differences between the background noise conditions were statistically significant. The greatest number of recall errors were committed in the unmasked speech condition, followed by the stationary masked condition, then the speech-shaped masking condition, and lastly, the silent condition. The results from this study suggest that masking sounds with a signal-to-noise ratio of anywhere from -6 to -9 $\mathrm{dB}$ helped participants receive the most working memory benefits. 
Conclusions: For optimal working memory benefits, type of masking noise, signal-to-noise ratio, and level of annoyance should be considered. Even if a condition is considered beneficial for one's working memory, an individual has to perceive the masking condition as bearable in order to use it.

Relevance to the current work: In the current work, masking conditions containing speech, as well as one stationary masking condition (i.e., pink noise) will be used. Similar to the results of this study, the current researchers anticipate that attention, and therefore overall speech and language behaviors, will differ according to which type of masking noise is used.

Smiljanic, R., \& Gilbert, R. C. (2017). Acoustics of clear and noise-adapted speech in children, young, and older adults. Journal of Speech, Language, and Hearing Research, 60(11), 3081-3096. https://doi.org/10.1044/2017_JSLHR-S-16-0130

Objective: This study focused on analyzing the acoustic and articulatory changes that occur when children, young adults, and older adults are instructed to speak clearly vs. speak loudly in response to noise.

Methods: Ten children (ages 11-12), 10 young adults (ages 18-29), and 10 older adults (ages 60-84) produced 60 sentences first in silence and then while listening to sixtalker babble speech played over headphones at $80 \mathrm{~dB}$. Participants were instructed to read half the sentences in a casual manner and the other half in a clear manner. Fo range, $F_{0}$ mean, rate of speech, intensity, and spectrum energy between 1-3 kHz were measured for each sample. A mixed effect linear regression was used with the variables of age, condition (noisy or quiet), and speaking style (casual or clear). 
Results: In both the noise-adapted speech and clear speech across all ages, speaking rate slowed down, the spectra became more balanced (i.e., more energy was located in the 1-3 kHz range), intensity increased, the vowel space became larger, and the harmonics-to-noise ratio increased. Speech produced in noise was associated with a raising of mean $\mathrm{F}_{0}$ and a decrease in jitter and shimmer. Clear speech was also associated with a raising of $\mathrm{F}_{0}$, as well as longer pauses. When compared to the children and younger adults, the older adults had the slowest rate of speech, the longest pauses, the smallest increase of mean $\mathrm{F}_{0}$, the least balanced spectra, and the lowest intensity while speaking clearly.

Conclusions: Although there are some similarities between the acoustic and articulatory adaptations made for noise-adapted speech and clear speech, the two types of speech are distinct. Additionally, the results from this study suggest that the extent of adaptations is associated with the age of the speaker.

Relevance to the current work: The majority of the current work's participants will most likely fall in the $60-84$ age category. Thus, it is beneficial to know specific acoustic and articulatory adaptations that this group may use when presented with noise.

Summers, V. W., Pisoni, D. B., Bernacki, R. H., Pedlow, R. I., \& Stokes, M. A (1988). Effects of noise on speech production: Acoustic and perceptual analyses. Journal of the Acoustical Society of America, 84(3), 917-928. https://doi.org/10.1121/1.396660

Objective: The purpose of this study was to examine the effects of masking on speech, specifically the acoustic and phonetic changes.

Methods: Two English-speaking males were instructed to read 15 Air Force vocabulary words (e.g., "threat") in a silent condition and with 80, 90, and $100 \mathrm{~dB}$ white 
noise playing over headphones. The stimulus words and the background conditions were given in a random order, and the two participants read the words in each of the conditions five times. Intensity, word duration, $\mathrm{F}_{0}$, spectral balance, and F1 and F2 were analyzed in each of the speech samples. ANOVA tests were performed to determine if acoustic measures differed significantly between background noise conditions.

Results: Intensity, word duration, $\mathrm{F}_{0}$, and spectral balance increased in the speech samples as the white noise got louder. For one participant, F1 also increased as the intensity of the white noise increased. Intensity, $\mathrm{F}_{0}$, and word duration adaptations from the $80 \mathrm{~dB}$ condition to the $100 \mathrm{~dB}$ condition were smaller than the researchers had anticipated (e.g., there was only a $2 \mathrm{~dB}$ increase in intensity between the two conditions, while previous research has found a $5 \mathrm{~dB}$ increase in speech intensity for every $10 \mathrm{~dB}$ increase in masking noise).

Conclusions: The results of the study suggest that acoustic/phonetic adaptations are made in noise, but that these adaptations may not increase linearly with the level of intensity of the masking noise; the authors pointed out that larger adaptations may have occurred at greater levels of intensity if the study sessions had been performed in an interactive setting (where external feedback to speak louder could be provided).

Relevance to the current work: Similar to this study, in the current work, various background noise conditions will be used. The results from this study support the current researchers' decision to learn more about speech adaptations in various conditions by differing the type of masking, rather than intensity of the masking. 
Wilson, S. M., Eriksson, D. K., Schneck, S. M., \& Lucanie, J. M. (2018). A quick aphasia battery for efficient, reliable, and multidimensional assessment of language function. PLoS ONE, 13(6), Article e0199469. https://doi.org/10.1371/journal.pone.0192773 Objective: The purpose of this paper was to describe the different sections of the Quick Aphasia Battery (QAB), an assessment designed to evaluate the language skills of PWA in 15-20 minutes. An additional purpose of this study was to assess the validity of the QAB.

Methods: The QAB was administered to the following groups: 1) acute stroke patients with aphasia (28 participants), 2) acute stroke patients without aphasia (25 participants), 3) chronic stroke patients with aphasia (16 participants), and 4) a control group (14 participants). The QAB was administered once to each of the acute and control participants, and three times to the chronic stroke patients. QAB results were recorded, transcribed, and scored. Correlations were then applied to the QAB results and previous Western Aphasia Battery (WAB) results.

Results: The 48 evaluations performed with the chronic stroke patients with aphasia were used for inter-rater reliability ratings and test-retest ratings. The inter-rater reliability for each of the QAB subtests ranged from .91 to .99. The test-retest ratings for non-comprehension subtests ranged from .90 to .98 , while the comprehension measures (word and sentence comprehension) ranged from .72 to .73. All of the QAB scores were used to calculate sensitivity and specificity measures, with sensitivity at .91 and specificity at .95. Concurrent validity was investigated; QAB sections were correlated anywhere from .79 to .95 with corresponding sections of the WAB $(p<.001)$. 
Conclusions: The QAB has fairly high sensitivity and specificity. In addition, the QAB has high concurrent validity with the WAB.

Relevance to the current work: To gather a language profile for each participant with aphasia, the QAB will be administered. 
APPENDIX B

Participant Consent Form

\section{Consent to be a Research Subject}

\section{Introduction}

This longitudinal research study is being conducted by Tyson Harmon, Ph.D., CCC-SLP and Dr. Christopher Dromey, Ph.D., CCC-SLP at Brigham Young University. The purposes of this study are to (1) determine the impact of background noise conditions on spoken language and (2) learn about the communication experiences of people recovering language after a stroke or brain injury from their own perspective. You were invited to participate because you had a stroke or other brain injury that affected your communication.

\section{Procedures}

Your participation in this study will involve a single evaluation session lasting $\mathbf{1 . 5}$ to $\mathbf{2}$ hours. During this session, you will be asked to complete a number of tests, retell stories in background noise conditions, and respond to some questionnaire and interview questions.

The tests, questionnaires, and interview will involve:

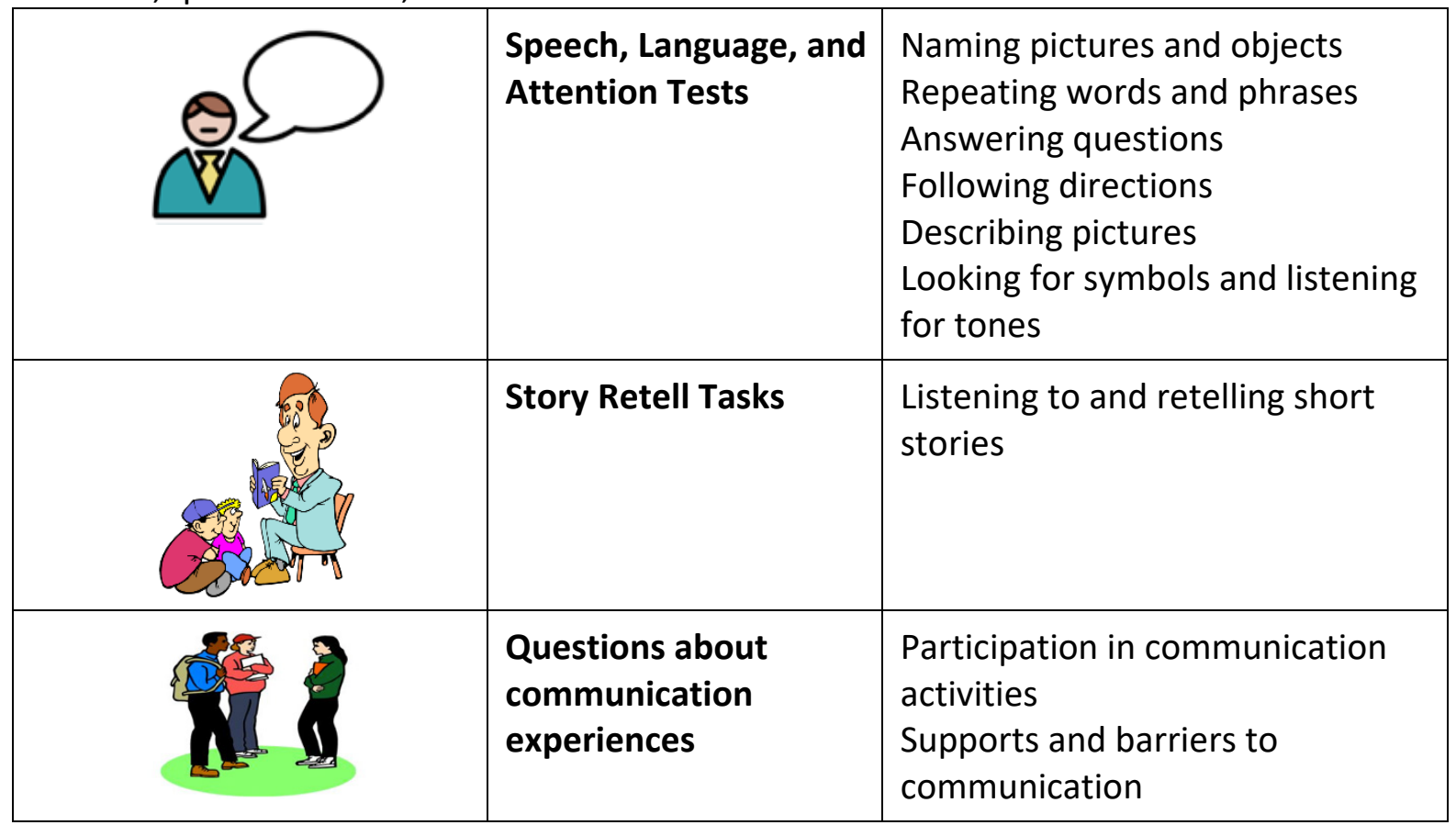

Several of these tests, questionnaires, and a brief interview will be audio or video recorded to check scores and complete more detailed analysis after the session. The session will be held on BYU campus (John Taylor Building room 110). 
As noted above, audio and video recordings will be obtained throughout the evaluation session. Please indicate what uses of these recordings you are willing to permit, by initialing next to the uses you agree to and signing at the end. This choice is completely up to you.

Yes N_ No Audio and/or video recordings can be studied by the research team for use in the research project.

Yes No Short excerpts of audio and/or video recordings can be used for scientific publications, conferences, or meetings.

Yes No Short excerpts of audio and/or video recordings can be shown in university classes.

\section{Risks/Discomforts}

Risks associated with this study are minimal. Because some of the test items may be difficult, you may become anxious or embarrassed. You might also become tired or frustrated. We will make every effort to be sure you are as comfortable as possible during the testing. You can take a break or discontinue your participation at any time. If the session is too long, the length and number of sessions can be changed according to your needs.

\section{Benefits}

Since this is not a treatment study, there is likely no direct benefit to you. However, your participation in this study will provide us with information that might generally improve assessment and treatment of people with communication impairments following stroke or brain injury.

\section{Confidentiality}

All data collected for the purposes of this study will be kept confidential and will only be reported without personally identifiable information.

You will be given a number that will identify you for this study. All data obtained from you will be associated with this number instead of your personally identifiable information. Any paper forms or test protocols will be kept in locked cabinets in a locked research lab at BYU. Any electronic forms or files (e.g., audio files) will be kept on a secured, password protected server. Only those directly involved with the research will have access to these data.

\section{Compensation}

You will receive $\$ 15.00$ cash after completing the session.

\section{Participation}

Participation in this research study is voluntary. You have the right to withdraw at any time or refuse to participate entirely. You do not have to be in this study to receive clinical services through the BYU Speech and Language Clinic. Choosing to not participate will not jeopardize your services at BYU or any other healthcare service you receive. 


\section{Questions about the Research}

If you have questions regarding this study, you may contact Tyson Harmon, Ph.D., CCC-SLP by phone at 801-422-1251 or email at tyson_harmon@byu.edu.

Questions about Your Rights as Research Participants

If you have questions regarding your rights as a research participant contact IRB Administrator at (801) 422-1461; A-285 ASB, Brigham Young University, Provo, UT 84602; irb@byu.edu.

\section{Statement of Consent}

I have read, understood, and received a copy of the above consent and desire of my own free will to participate in this study.

Name (Printed):

Date: 